\title{
Renormalization of a generalized supersymmetric version of the maximal Abelian gauge
}

\author{
M. A. L. Capri, ${ }^{1, *}$ R. C. Terin, ${ }^{1,2, \dagger}$ and H. C. Toledo ${ }^{1, \$}$ \\ ${ }^{1}$ Departamento de Física Teórica, Instituto de Física, Universidade do Estado do Rio de Janeiro, \\ UERJ, Rua São Francisco Xavier 524, 20550-013, Rio de Janeiro, RJ, Brazil \\ ${ }^{2}$ Sorbonne Université, CNRS, Laboratoire de Physique Théorique de la Matière Condensée, \\ LPTMC, F-75005 Paris, France
}

(Received 3 December 2018; published 31 January 2019)

\begin{abstract}
In this work we present an algebraic proof of the renormazibility of the super-Yang-Mills action quantized in a generalized supersymmetric version of the maximal Abelian gauge. The main point stated here is that the generalized gauge depends on a set of infinity gauge parameters in order to take into account all possible composite operators emerging from the dimensionless character of the vector superfield. At the end, after the removal of all ultraviolet divergences, it is possible to specify values to the gauge parameters in order to return to the original supersymmetric maximal Abelian gauge, first presented in Phys. Rev. D 91, 125017 (2015).
\end{abstract}

DOI: $10.1103 /$ PhysRevD.99.025015

\section{INTRODUCTION}

In the understanding of the quark confinement mechanism, some formulations of the Yang-Mills theory in specific gauge conditions, like the Landau gauge, the maximal Abelian gauge, the Curci-Ferrari gauge, etc., are explored. In particular, the maximal Abelian gauge permits us to approach the notion of Abelian projection, one of the main ideas regarding quark confinement [1]. Here, the emergence of the magnetic monopoles, provided by the Abelian degrees, can be understood as a confinement mechanism. However, in the supersymmetric scenario, the subject of confinement leads us directly to the AdS/CFT correspondence where there would be a duality between a ten-dimensional lowtemperature string theory with the strong couplings of a $N=4$ theory of super-Yang-Mills in four dimensions [2].

In fact, supersymmetric theories at finite temperature can reveal fundamental properties, similar to those of weak interactions in a plasma of quarks and gluons. Thus, it may be possible to study the transition from the deconfinement phase in an analogous way to the nonsupersymmetric case, i.e., to analyze phase transitions due to the emergence of singularities in the Abelian sector of a super-Yang-Mills theory.In this work, our focus is on the proof of the

\footnotetext{
*caprimarcio@gmail.com

†rodrigoterin3003@gmail.com

"henriqcouto@gmail.com
}

Published by the American Physical Society under the terms of the Creative Commons Attribution 4.0 International license. Further distribution of this work must maintain attribution to the author(s) and the published article's title, journal citation, and DOI. Funded by SCOAP. renormazibility of the super-Yang-Mills theory in the supersymmetric maximal Abelian gauge, as proposed in [3]. However, since the vector superfield, $V(x, \theta, \bar{\theta})$, is dimensionless $^{1}$ the construction of the most general counterterm, which cancels all divergences of the theory, becomes complicated because there are infinite insertions depending on the vector superfield. Therefore, the version of the maximal Abelian gauge presented in [3] is not unique; it is actually the simplest one, and the correct definition of this gauge in superspace seems to be ambiguous. The solution for this problem is a generalization of the gauge-fixing condition in order to include all ambiguities in the definition of the gauge. Namely,

$$
\begin{gathered}
\overline{\mathcal{D}}^{2} \mathcal{D}^{2} V^{i}=0 \rightarrow \overline{\mathcal{D}}^{2} \mathcal{D}^{2} \omega^{i}(V)=0, \\
\overline{\mathcal{D}}^{2} \mathcal{D}^{2}\left(V^{a}-\frac{i}{2} f^{a b i} V^{i} V^{b}\right)=0 \rightarrow \overline{\mathcal{D}}^{2} \mathcal{D}^{2} \omega^{a}(V)=0,
\end{gathered}
$$

where the expressions in the lhs are the original conditions of the maximal Abelian gauge in superspace introduced in [3], and $\omega^{a}(V)$ and $\omega^{i}(V)$ are general power series in $V$ obeying some symmetry criteria that will be clear later in Sec. II D. Also, the index conventions will be clarified in Sec. II A and in the Appendix, but at the moment it is sufficient to say that the index $i$ is related to the Abelian components of the internal symmetry group, the $\mathrm{SU}(n)$ group, and the indices $\{a, b\}$ to the non-Abelian ones. One advantage of this method lies in the fact that it defines a general class of nonlinear gauges depending on a set of

\footnotetext{
${ }^{1}$ See Table I and the discussion in Sec. II D.
} 
gauge parameters that can be chosen in a suitable way after the renormalization procedure, or, in other words, after the removal of the ultraviolet divergences of the theory. Then, the supersymmetric version of the maximal Abelian gauge is viewed here as a particular case of this general gauge. Actually, even the Landau gauge can be classified into this general class [4].

The paper is organized as follows. Section II is subdivided into five subsections in which we give a brief review of the supersymmetric extension of the maximal Abelian gauge, as presented in [3], and how it can be generalized. The classical starting point action is also defined in this section and its rich symmetry content is meticulously discussed. In Sec. III, we perform the proof of renormalizability of the theory following the algebraic renormalization setup outlined in Refs. [4-12]. ${ }^{2}$ The renormalization factors (the "famous" $Z$ factors) of fields, sources, and parameters of the theory are obtained, making clear that the renormalization of the vector superfield is nonlinear and its components (the Abelian and non-Abelian ones) are mixed in quantum corrections into a matricial renormalization. In Sec. IV, we conclude this work with some final discussions and perspectives for future works. Finally, in the Appendix, we display, for the sake of the reader, a review on the maximal Abelian gauge for ordinary $\mathrm{SU}(n)$ Yang-Mills theory and some relevant notations and conventions are properly defined.

\section{THE SUPERSYMMETRIC MAXIMAL ABELIAN GAUGE}

\section{A. The simplest formulation}

In this section we perform a brief review on the supersymmetric formulation of the maximal Abelian gauge, first presented in [3]. ${ }^{3}$ The main characteristic of this gauge is to explicitly split the Abelian and non-Abelian sectors of the gauge symmetry group. In the $\mathrm{SU}(n)$ group one can decompose the vector superfield $V(x, \theta, \bar{\theta})$ in terms of the group generators as

$$
\begin{aligned}
V(x, \theta, \bar{\theta}) & =\sum_{A=1}^{n^{2}-1} V^{A}(x, \theta, \bar{\theta}) T^{A} \\
& =\sum_{a=1}^{n(n-1)} V^{a}(x, \theta, \bar{\theta}) T^{a}+\sum_{i=1}^{n-1} V^{i}(x, \theta, \bar{\theta}) T^{i},
\end{aligned}
$$

\footnotetext{
${ }^{2}$ References [5-7] are standard references on the procedure of the algebraic renormalization, while Refs. [4,8-12] relate to the renormalization in the presence of a dimensionless vector superfield in the Landau and linear covariant gauges. The problem of dealing with the renormalization of a theory with dimensionless fields is also approached in Refs. [13,14] in the case of a Stueckelberg-like field.

${ }^{3}$ See also the Appendix for a review of the maximal Abelian gauge for the ordinary Yang-Mills theory in the $\mathrm{SU}(n)$ group.
}

where $T^{A}$ stands by the $n^{2}-1$ group generators of $\mathrm{SU}(n)$, which can be split into the $n(n-1)$ off-diagonal generators, $T^{a}$, and the $n-1$ diagonal generators, $T^{i}$, which form an Abelian subgroup of $\mathrm{SU}(n)$ also known as the Cartan subgroup. Also, we have adopted here capital letters $\{A, B, C, \ldots\}$, running from 1 to $n^{2}-1$, for the full $\mathrm{SU}(n)$ group; the labels $\{a, b, c, d, e, \ldots\}$, running from 1 to $n(n-1)$, for the off-diagonal sector; and the indices $\{i, j, k, l, \ldots\}$, running from 1 to $n-1$, for the Abelian sector. Of course, we will assume, from now on, the Einstein summation convention for repeated indices. ${ }^{4}$

In order to make clear our notations and conventions, let us display here the vector superfield in terms of its components:

$$
\begin{aligned}
V^{A}= & C^{A}(x)+\theta^{\alpha} \chi_{\alpha}^{A}(x)+\bar{\theta}_{\dot{\alpha}} \bar{\chi}^{A \dot{\alpha}}(x) \\
& +\frac{1}{2} \theta^{2} M^{A}(x)+\frac{1}{2} \bar{\theta}^{2} \bar{M}^{A}(x)+2 \theta^{\alpha} \sigma_{\alpha \dot{\alpha}}^{\mu} \bar{\theta}^{\dot{\alpha}} A_{\mu}^{A}(x) \\
& +\frac{1}{2} \bar{\theta}^{2} \theta^{\alpha} \lambda_{\alpha}^{A}(x)+\frac{1}{2} \theta^{2} \bar{\theta}_{\dot{\alpha}} \bar{\lambda}^{A \dot{\alpha}}(x)+\frac{1}{4} \theta^{2} \bar{\theta}^{2} \mathfrak{D} A(x),
\end{aligned}
$$

where $\theta^{\alpha}(\alpha=1,2)$ and $\bar{\theta}_{\dot{\alpha}}(\dot{\alpha}=1, \dot{2})$ are the fermionic coordinates of the superspace; and $\left\{C, \chi_{\alpha}, \bar{\chi}_{\dot{\alpha}}, M, \bar{M}, A_{\mu}, \lambda_{\alpha}\right.$, $\left.\bar{\lambda}_{\dot{\alpha}}, \mathfrak{D}\right\}$ the superfield components in the adjoint representation of the $\mathrm{SU}(n)$ group. 5

It is well known that in the quantization of a gauge field theory (being supersymmetric or not) an additional condition (or a constraint, or a gauge-fixing condition) for the gauge field needs to be implemented. Following [3], such additional condition can be chosen as

$$
\begin{gathered}
\overline{\mathcal{D}}^{2} \mathcal{D}^{2}\left(V^{a}-\frac{i}{2} f^{a b i} V^{i} V^{b}\right)=0, \\
\overline{\mathcal{D}}^{2} \mathcal{D}^{2} V^{i}=0
\end{gathered}
$$

where $\mathcal{D}_{\alpha}$ and $\overline{\mathcal{D}}_{\dot{\alpha}}$ are, respectively, the chiral and antichiral covariant derivatives, given by

$$
\begin{gathered}
\mathcal{D}_{\alpha}=\frac{\partial}{\partial \theta^{\alpha}}-i \sigma_{\alpha \dot{\alpha}}^{\mu} \bar{\theta}^{\dot{\alpha}} \partial_{\mu}, \\
\overline{\mathcal{D}}_{\dot{\alpha}}=-\frac{\partial}{\partial \bar{\theta}^{\dot{\alpha}}}-i \theta^{\alpha} \sigma_{\alpha \dot{\alpha}}^{\mu} \partial_{\mu},
\end{gathered}
$$

and $f^{a b i}$ represents one of the possible types of structure constants appearing in the algebra associated with the $\mathrm{SU}(n)$ group; see details in the Appendix. Considering the constraints (5) and (6) written in terms of the components of the superfield and, together with the so-called Wess-Zumino gauge, i.e., taking the lower components

\footnotetext{
${ }^{4}$ The algebra of the generators and some useful identities can be found in the Appendix.

${ }^{5} \mathrm{We}$ also have in Eq. (4) that $\theta^{2}=\theta^{\alpha} \theta_{\alpha}, \bar{\theta}^{2}=\bar{\theta}_{\dot{\alpha}} \bar{\theta}^{\dot{\alpha}}$, and $\sigma^{\mu}=\left(\mathbf{1}, \sigma_{1}, \sigma_{2}, \sigma_{3}\right)$, with 1 the identity matrix of order 2 and $\left(\sigma_{1}, \sigma_{2}, \sigma_{3}\right)$ the usual Pauli matrices.
} 
$\{C, \chi, \bar{\chi}, M, \bar{M}\}$ equal to zero, it is possible to obtain the usual maximal Abelian gauge [3]:

$$
\begin{gathered}
\partial_{\mu} A^{a \mu}=f^{a b i} A_{\mu}^{i} A^{b \mu}, \\
\partial_{\mu} A^{i \mu}=0 .
\end{gathered}
$$

As we shall see later, the superfield $V(x, \theta, \bar{\theta})$ is dimensionless. Therefore, the off-diagonal gauge-fixing condition (5) is the simplest way to define the supersymmetric version of the maximal Abelian gauge, while the diagonal condition (6) corresponds to a Landau-like gauge. Thus, we will name the gauge defined by Eqs. (5) and (6) as simplest supermaximal Abelian gauge (SSMAG). In Sec. II D, we will discuss a generalized version of SSMAG that we will call the generalized supermaximal Abelian gauge (GSMAG).

\section{B. The Faddeev-Popov quantization and BSRT symmetry}

Since we have defined the gauge conditions of the SSMAG, Eqs. (5) and (6), we are able to carry out the Faddeev-Popov quantization procedure [12,15-17]. First of all, let us start with the super-Yang-Mills (SYM) action:

$$
\begin{aligned}
S_{\mathrm{SYM}} & =-\frac{1}{64 g^{2}} \operatorname{tr} \int d^{4} x d^{2} \theta \mathcal{W}^{\alpha} \mathcal{W}_{\alpha}+\text { c.c. } \\
& =-\frac{1}{128 g^{2}} \int d^{4} x d^{2} \theta W^{A \alpha} W_{\alpha}^{A}+\text { c.c. }
\end{aligned}
$$

where $g$ is the coupling constant and $\mathcal{W}_{\alpha}$ the chiral-field strength, given by

$$
\mathcal{W}_{\alpha}=W_{\alpha}^{A} T^{A}=\overline{\mathcal{D}}^{2}\left(e^{-V} \mathcal{D}_{\alpha} e^{V}\right)
$$

It can be checked that the action (11) is invariant by the following infinitesimal gauge transformations:

$$
\begin{aligned}
V \rightarrow V^{\prime}= & V+\delta V \\
\delta V= & \frac{i}{2} L_{V}(\Lambda+\bar{\Lambda})+\frac{i}{2}\left[L_{V} \operatorname{coth}\left(\frac{1}{2} L_{V}\right)\right](\Lambda-\bar{\Lambda}) \\
= & i(\Lambda-\bar{\Lambda})+\frac{1}{2}[V, \Lambda+\bar{\Lambda}] \\
& +\frac{i}{12}[V,[V, \Lambda-\bar{\Lambda}]]+\mathcal{O}\left(V^{3}\right)
\end{aligned}
$$

where $L_{V} \bullet=[V, \bullet]$ and $\Lambda=\Lambda^{A} T^{A}$ are chiral infinitesimal superfields, while $\bar{\Lambda}=\bar{\Lambda}^{A} T^{A}$ are antichiral superfields. As a gauge theory, the correct quantization ${ }^{6}$ needs the

\footnotetext{
${ }^{6}$ Actually, the correct quantization of a gauge theory is an open problem until now. The Faddeev-Popov method is considered to be correct only at the perturbative level, but, at the nonperturbative level, other effects, such as the Gribov ambiguity problem, show up and have to be taken into account. In this work we will restrict ourselves to the Faddeev-Popov quantization method.
}

implementation of a gauge-fixing condition. The FaddeevPopov method corresponds to a way of introducing a constraint in the functional integral for a gauge theory. In this method the SYM action needs to be supplemented, in the Feynman path integrals, by a term including such a constraint. Then, according to the Faddeev-Popov method, the SYM action is replaced by

$$
S_{\mathrm{FP}}=S_{\mathrm{SYM}}+S_{\mathrm{gf}},
$$

where $S_{\mathrm{gf}}$ is the gauge-fixing action. In our case, the gaugefixing action for the SSMAG is, following [3], given by

$$
\begin{aligned}
S_{\mathrm{gf}}= & \frac{1}{8} \int d^{4} x d^{2} \theta\left[B^{a} \overline{\mathcal{D}}^{2} \mathcal{D}^{2}\left(V^{a}-\frac{i}{2} f^{a b i} V^{i} V^{b}\right)\right. \\
& \left.+B^{i} \overline{\mathcal{D}}^{2} \mathcal{D}^{2} V^{i}\right]+ \text { "ghost terms" }+ \text { c.c. }
\end{aligned}
$$

The action above needs several explanations. First, the fields $B^{a}$ and $B^{i}$ play the role of Lagrange multipliers enforcing the SSMAG conditions for the off-diagonal and diagonal sectors, respectively. It is immediately checked that their classical equations of motion coincide with the constraints (5) and (6). Also, it is easy to notice that they are chiral superfields. Then, in the complex conjugated part, standing by "c.c.," the antichiral superfields $\bar{B}^{a}$ and $\bar{B}^{i}$ must appear. The term "ghost terms" represents here all terms involving the well-known Faddeev-Popov ghost fields. In order to avoid any confusion, before presenting this term explicitly, let us display here the four sets of ghosts that we have to deal with:

(i) The off-diagonal chiral ghosts $\left\{c^{a}, c_{\star}^{a}\right\}$;

(ii) The off-diagonal antichiral ghosts $\left\{\bar{c}^{a}, \bar{c}_{\star}^{a}\right\}$;

(iii) The diagonal chiral ghosts $\left\{c^{i}, c_{\star}^{i}\right\}$;

(iv) The diagonal antichiral ghosts $\left\{\bar{c}^{a}, \bar{c}_{\star}^{a}\right\}$.

In each set listed above, the subscribed symbol " $\star$ " indicates the antighost and the bar indicates the antichiral character of the field. For example, $c^{a}$ is the off-diagonal chiral ghost, $c_{\star}^{i}$ is the diagonal chiral antighost, and $\bar{c}_{\star}^{a}$ is the off-diagonal antichiral antighost. Another point that must be explained here is that, although the gauge invariance has been lost in the gauge-fixing procedure, the Faddeev-Popov action $S_{\mathrm{FP}}$ is left invariant by a set of transformations, the so-called BecchiRouet-Stora-Tyutin (BRST) transformations, listed below:

(i) Transformations of the components of the vector superfield $V(x, \theta, \bar{\theta})$ :

$$
\begin{aligned}
s V^{a}= & i\left(c^{a}-\bar{c}^{a}\right)-\frac{1}{2} f^{a b c} V^{b}\left(c^{c}+\bar{c}^{c}\right) \\
& -\frac{1}{2} f^{a b i} V^{b}\left(c^{i}+\bar{c}^{i}\right)+\frac{1}{2} f^{a b c} V^{i}\left(c^{b}+\bar{c}^{b}\right) \\
& +\mathcal{O}\left(V^{2}\right), \\
s V^{i}= & i\left(c^{i}-\bar{c}^{i}\right)-\frac{1}{2} f^{a b i} V^{a}\left(c^{b}+\bar{c}^{b}\right)+\mathcal{O}\left(V^{2}\right) ;
\end{aligned}
$$


(ii) Transformations of the components of chiral superfields $\left\{c, c_{\star}, B\right\}$ :

$$
\begin{array}{ll}
s c^{a}=f^{a b i} c^{b} c^{i}+\frac{1}{2} f^{a b c} c^{b} c^{c}, & s c^{i}=\frac{1}{2} f^{a b i} c^{a} c^{b}, \\
s c_{\star}^{a} & =B^{a}, \quad s c_{\star}^{i}=B^{i}, \\
s B^{a} & =0, \quad s B^{i}=0 ;
\end{array}
$$

(iii) Transformations of the components of the antichiral superfields $\left\{\bar{c}, \bar{c}_{\star}, \bar{B}\right\}$ :

$$
\begin{array}{ll}
s \bar{c}^{a}=f^{a b i} \bar{c}^{b} \bar{c}^{i}+\frac{1}{2} f^{a b c} \bar{c}^{b} \bar{c}^{c}, \quad s \bar{c}^{i}=\frac{1}{2} f^{a b i} \bar{c}^{a} \bar{c}^{b}, \\
s \bar{c}_{\star}^{a}=\bar{B}^{a}, \quad s \bar{c}_{\star}^{i}=\bar{B}^{i}, \\
s \bar{B}^{a}=0, \quad s \bar{B}^{i}=0 .
\end{array}
$$

As one can see, the BRST transformations of the vector superfield are similar to the infinitesimal gauge transformations (13), just replacing the infinitesimal gauge parameters $\{\Lambda, \bar{\Lambda}\}$ by the ghosts $\{c, \bar{c}\}$. Therefore, the SYM action is automatically invariant by the set of transformations (16). Also, the BRST operator, $s$, is nilpotent, i.e., $s^{2}=0$, and, thanks to this remarkable property, one can finally write the gauge-fixing term as a full BRST variation:

$$
\begin{aligned}
S_{\mathrm{gf}}= & \frac{1}{8} s \int d V\left[c_{\star}^{a} \mathcal{D}^{2}\left(V^{a}-\frac{i}{2} f^{a b i} V^{i} V^{b}\right)+c_{\star}^{i} \mathcal{D}^{2} V^{i}\right. \\
& \left.+\bar{c}_{\star}^{a} \overline{\mathcal{D}}^{2}\left(V^{a}+\frac{i}{2} f^{a b i} V^{i} V^{b}\right)+\bar{c}_{\star}^{i} \overline{\mathcal{D}}^{2} V^{i}\right] \\
= & \frac{1}{8} \int d V\left[B^{a} \mathcal{D}^{2}\left(V^{a}-\frac{i}{2} f^{a b i} V^{i} V^{b}\right)+B^{i} \mathcal{D}^{2} V^{i}\right. \\
& \left.+\bar{B}^{a} \overline{\mathcal{D}}^{2}\left(V^{a}+\frac{i}{2} f^{a b i} V^{i} V^{b}\right)+\bar{B}^{i} \overline{\mathcal{D}}^{2} V^{i}\right] \\
& -\frac{1}{8} \int d V\left[c_{\star}^{a} \mathcal{D}^{2} s\left(V^{a}-\frac{i}{2} f^{a b i} V^{i} V^{b}\right)+c_{\star}^{i} \mathcal{D}^{2} s V^{i}\right. \\
& \left.+\bar{c}_{\star}^{a} \overline{\mathcal{D}}^{2} s\left(V^{a}+\frac{i}{2} f^{a b i} V^{i} V^{b}\right)+\bar{c}_{\star}^{i} \overline{\mathcal{D}}^{2} s V^{i}\right]
\end{aligned}
$$

where $d V \equiv d^{4} x d^{2} \theta d^{2} \bar{\theta}$ is the superspace element volume. The action above is evidently invariant due to the nilpotency property and thus the Faddeev-Popov action (14) is BRST invariant. Summarizing our current situation, we have at our disposal the BRST invariant action (14), representing the $N=1 \mathrm{SYM}$ theory for a $\mathrm{SU}(n)$ group quantized in the socalled SSMAG. The next step would be the study of the renormalizability of this model. In order to achieve this aim, let us first study the symmetry content and the Ward identities of the model.

\section{Local composite operator formalism and the Ward identities}

In this section we would like to study the symmetry content of the action (14). We already known that action (14) is BRST invariant and the BRST transformations are nonlinear. In order to deal with such nonlinear symmetry, and other possible nonlinear identities of the model, we need to make use of the local composite operator formalism [7]. For this purpose, let us consider the following action:

$$
S=S_{\mathrm{FP}}+S_{\mathrm{ext}}
$$

The external action $S_{\text {ext }}$ is a term depending on external sources coupled to some local composite operators. More specifically, we have

$$
\begin{aligned}
S_{\mathrm{ext}}[\Omega, L, \bar{L}, R, P]= & \int d V\left[\Omega^{a}\left(s V^{a}\right)+\Omega^{i}\left(s V^{i}\right)\right] \\
& +\int d^{4} x d^{2} \theta\left[L^{a}\left(s c^{a}\right)+L^{i}\left(s c^{i}\right)\right] \\
& +\int d^{4} x d^{2} \bar{\theta}\left[\bar{L}^{a}\left(s \bar{c}^{a}\right)+\bar{L}^{i}\left(s \bar{c}^{i}\right)\right] \\
& +\int d V P^{a}\left(f^{a b i} V^{i} V^{b}\right) \\
& -\int d V R^{a} s\left(f^{a b i} V^{i} V^{b}\right),
\end{aligned}
$$

where the BRST invariance of the external term is guaranteed by the BRST transformations of the external sources below:

$$
\begin{aligned}
& s \Omega^{a}=0, \quad s \Omega^{i}=0, \quad s L^{a}=0, \quad s \bar{L}^{a}=0, \\
& s L^{i}=0, \quad s \bar{L}^{i}=0, \quad s R^{a}=P^{a}, \quad s P^{a}=0 .
\end{aligned}
$$

Notice that the sources $\{\Omega, L, \bar{L}\}$ are coupled to the nonlinear BRST transformations, while the sources $P^{a}$ and $R^{a}$ are coupled to the composite operator $f^{a b i} V^{i} V^{b}$ and its BRST transformation, respectively. Furthermore, the sources $\{R, P\}$ form the so-called BRST doublet and the last two terms of (21) can be written as an exact BRST variation. In fact, the external term can be completely written as a BRST variation:

$$
\begin{aligned}
S_{\mathrm{ext}}[\Omega, L, \bar{L}, R, P]= & s\left[\int d V\left(-\Omega^{a} V^{a}-\Omega^{i} V^{i}\right)\right. \\
& +\int d^{4} x d^{2} \theta\left(L^{a} c^{a}+L^{i} c^{i}\right) \\
& +\int d^{4} x d^{2} \bar{\theta}\left(\bar{L}^{a} \bar{c}^{a}+\bar{L}^{i} \bar{c}^{i}\right) \\
& \left.+\int d V f^{a b i} R^{a} V^{i} V^{b}\right] .
\end{aligned}
$$


TABLE I. Mass dimension $d$, ghost number $g \#$, and $\mathcal{R}$-weights $n$ of the fields, sources, covariant derivatives, etc.

\begin{tabular}{lllrlrlrrrrrrrrrrr}
\hline \hline & $V$ & $c$ & $c_{\star}$ & $\bar{c}$ & $\bar{c}_{\star}$ & $B$ & $\bar{B}$ & $\Omega$ & $L$ & $\bar{L}$ & $\mathcal{D}$ & $\overline{\mathcal{D}}$ & $\theta$ & $\bar{\theta}$ & $R$ & $P$ & $s$ \\
\hline$d$ & 0 & 0 & 1 & 0 & 1 & 1 & 1 & 2 & 3 & 3 & $1 / 2$ & $1 / 2$ & $-1 / 2$ & $-1 / 2$ & 2 & 2 & 0 \\
$g \#$ & 0 & 1 & -1 & 1 & -1 & 0 & 0 & -1 & -2 & -2 & 0 & 0 & 0 & 0 & -1 & 0 & 1 \\
$n$ & 0 & 0 & 2 & 0 & -2 & 2 & -2 & 0 & 2 & -2 & -1 & 1 & 1 & -1 & 0 & 0 & 0 \\
\hline \hline
\end{tabular}

The external sources are introduced here as a mathematical tool that allow us to define some important Green functions of the model and to write in a well-defined manner the nonlinear Ward identities of the model. As these sources vanish, the action $S$, Eq. (20), coincides with $S_{\mathrm{FP}}$, Eq. (14). Therefore, action (20) is suitable to study the symmetry content in terms of Ward identities, including the nonlinear ones. As a last remark before discussing the Ward identities, we would like to call attention to the fact that the BRST operator, $s$, and the Faddeev-Popov ghosts are Grassmann variables and carry a quantum number named the "ghost number" (g\#). In Table I we displayed the quantum numbers of the fields and sources of the theory, including the mass dimensions. Notice that the sources with odd ghost number, as $\Omega$ and $R$, are anticommuting, while the sources with even ghost number, as $L, \bar{L}$, and $P$, are commuting. ${ }^{7}$

Now we are able to present the set of Ward identities enjoyed by the action (20). These identities represent the set of all symmetries of the theory being fundamental for the proof of its renormalizability.

\section{The Slavnov-Taylor identity}

The BRST symmetry can be written as a functional identity as follows:

$$
\begin{aligned}
\mathcal{B}(S): & =\int d V\left(\frac{\delta S}{\delta \Omega^{a}} \frac{\delta S}{\delta V^{a}}+\frac{\delta S}{\delta \Omega^{i}} \frac{\delta S}{\delta V^{i}}+P^{a} \frac{\delta S}{\delta R^{a}}\right) \\
& +\int d^{4} x d^{2} \theta\left(\frac{\delta S}{\delta L^{a}} \frac{\delta S}{\delta c^{a}}+\frac{\delta S}{\delta L^{i}} \frac{\delta S}{\delta c^{i}}+B^{a} \frac{\delta S}{\delta c_{\star}^{a}}+B^{i} \frac{\delta S}{\delta c_{\star}^{i}}\right) \\
& +\int d^{4} x d^{2} \bar{\theta}\left(\frac{\delta S}{\delta \bar{L}^{a}} \frac{\delta S}{\delta \bar{c}^{a}}+\frac{\delta \Sigma}{\delta \bar{L}^{i}} \frac{\delta S}{\delta \bar{c}^{i}}+\bar{B}^{a} \frac{\delta S}{\delta \bar{c}_{\star}^{a}}+\bar{B}^{i} \frac{\delta S}{\delta \bar{c}_{\star}^{i}}\right) \\
= & 0 .
\end{aligned}
$$

\section{The diagonal gauge-fixing equations}

The classical equations of motion of the diagonal Lagrange multipliers $B^{i}$ and $\bar{B}^{i}$, being linear in the fields, can be recognized as valid equations of the so-called quantum action principle (QAP) [7]. Namely,

\footnotetext{
${ }^{7}$ In general, if the combination $2 d+(g \#)$, with $d$ being the mass dimension, is an even number, the corresponding object (a field, a source, a parameter, or an operator) is commuting. Otherwise it is anticommuting.
}

$$
\frac{\delta S}{\delta B^{i}}=\frac{1}{8} \overline{\mathcal{D}}^{2} \mathcal{D}^{2} V^{i}, \quad \frac{\delta S}{\delta \bar{B}^{i}}=\frac{1}{8} \mathcal{D}^{2} \overline{\mathcal{D}}^{2} V^{i}
$$

\section{The off-diagonal gauge-fixing equations}

In contrast with the diagonal equations of motion of the Lagrange multipliers $\left\{B^{i}, \bar{B}^{i}\right\}$, the equations of motion of the off-diagonal Lagrange multipliers $\left\{B^{a}, \bar{B}^{a}\right\}$ are nonlinear. It is a direct consequence of the nonlinearity of the SSMAG. However, with the help of the insertion of the local composite operator $f^{a b i} V^{i} V^{b}$ we can write the equations of motion of the fields $\left\{B^{a}, \bar{B}^{a}\right\}$ as the following functional identities:

$$
\begin{aligned}
& \frac{\delta S}{\delta B^{a}}+\frac{i}{16} \overline{\mathcal{D}}^{2} \mathcal{D}^{2} \frac{\delta S}{\delta P^{a}}=\frac{1}{8} \overline{\mathcal{D}}^{2} \mathcal{D}^{2} V^{a}, \\
& \frac{\delta S}{\delta \bar{B}^{a}}-\frac{i}{16} \mathcal{D}^{2} \overline{\mathcal{D}}^{2} \frac{\delta S}{\delta P^{a}}=\frac{1}{8} \mathcal{D}^{2} \overline{\mathcal{D}}^{2} V^{a} .
\end{aligned}
$$

\section{The diagonal antighost equations}

From the diagonal antighost equations the following identities can be obtained ${ }^{8}$ :

$$
\frac{\delta S}{\delta c_{\star}^{i}}+\frac{1}{8} \overline{\mathcal{D}}^{2} \mathcal{D}^{2} \frac{\delta S}{\delta \Omega^{i}}=0, \quad \frac{\delta S}{\delta \bar{c}_{\star}^{i}}+\frac{1}{8} \mathcal{D}^{2} \overline{\mathcal{D}}^{2} \frac{\delta S}{\delta \Omega^{i}}=0 .
$$

\section{The off-diagonal antighost equations}

$$
\begin{aligned}
& \frac{\delta S}{\delta c_{\star}^{a}}+\frac{1}{8} \overline{\mathcal{D}}^{2} \mathcal{D}^{2} \frac{\delta S}{\delta \Omega^{a}}+\frac{i}{16} \overline{\mathcal{D}}^{2} \mathcal{D}^{2} \frac{\delta S}{\delta R^{a}}=0, \\
& \frac{\delta S}{\delta \bar{c}_{\star}^{a}}+\frac{1}{8} \mathcal{D}^{2} \overline{\mathcal{D}}^{2} \frac{\delta S}{\delta \Omega^{a}}-\frac{i}{16} \mathcal{D}^{2} \overline{\mathcal{D}}^{2} \frac{\delta S}{\delta R^{a}}=0 .
\end{aligned}
$$

Like the off-diagonal gauge-fixing equations, these identities are only possible in the presence of the composite

\footnotetext{
${ }^{8}$ Some authors call these equations the ghost equations. In our nomenclature, however, we have decided to name the equations obtained from the functional derivatives of the antighost fields (chiral or antichiral) as "antighost equations" and the identities obtained from the functional derivatives of the ghost fields as "ghost equations."
} 
operator $f^{a b i} V^{i} V^{b}$ and its BRST variation. It is important to remark here that in the nonsupersymmetric version of the MAG (see the Appendix), the analogous identities cannot be recovered by introducing any composite operators. ${ }^{9,10}$ Therefore, it seems to be a property of the superspace formulation.

\section{The $\mathcal{R}$-invariance}

$$
\begin{aligned}
\mathcal{R}(S):= & \sum_{X \in\{V, \Omega, R, P\}} \int d V\left(\delta_{\mathcal{R}} X\right) \frac{\delta S}{\delta X} \\
& +\sum_{Y \in\left\{c, c_{\star}, B, L\right\}} \int d^{4} x d^{2} \theta\left(\delta_{\mathcal{R}} Y\right) \frac{\delta S}{\delta Y} \\
& +\sum_{\bar{Y} \in\left\{\bar{c}, \bar{c}_{\star}, \bar{B}, \bar{L}\right\}} \int d^{4} x d^{2} \bar{\theta}\left(\delta_{\mathcal{R}} \bar{Y}\right) \frac{\delta S}{\delta \bar{Y}} \\
= & 0 .
\end{aligned}
$$

The $\mathcal{R}$-variations $\left(\delta_{\mathcal{R}} Z\right)$, with $Z$ being any superfield of the theory, are given by

$$
\delta_{\mathcal{R}} Z=i\left(n_{Z}+\theta^{\alpha} \frac{\partial}{\partial \theta^{\alpha}}-\bar{\theta}^{\dot{\alpha}} \frac{\partial}{\partial \bar{\theta}^{\dot{\alpha}}}\right) Z,
$$

with $n_{Z}$ being the so-called " $\mathcal{R}$-weight" of the respective superfield $Z$. The $\mathcal{R}$-weights of all objects present in the theory (fields, sources, covariant derivatives, etc.) are displayed in Table I.

\section{The diagonal rigid invariance}

$$
\begin{aligned}
\mathcal{W}^{i}(S):= & \sum_{X \in\{V, \Omega, R, P\}} \int d V f^{a b i} X^{a} \frac{\delta S}{\delta X^{b}} \\
& +\sum_{Y \in\left\{c, c_{\star}, B, L\right\}} \int d^{4} x d^{2} \theta f^{a b i} Y^{a} \frac{\delta S}{\delta Y^{b}} \\
& +\sum_{\bar{Y} \in\left\{\bar{c}, \bar{c}_{\star}, \bar{B}, \bar{L}\right\}} \int d^{4} x d^{2} \bar{\theta} f^{a b i} \bar{Y}^{a} \frac{\delta S}{\delta \bar{Y}^{b}} \\
= & 0 .
\end{aligned}
$$

Notice that the diagonal rigid symmetry involves transformations only in the off-diagonal components of fields and sources. Also, this symmetry is a consequence of the split of the diagonal and off-diagonal components of the group, which is the main characteristic of the SSMAG,

\footnotetext{
${ }^{9}$ Actually they can be recovered but they are completely innocuous.

${ }^{10} \mathrm{~A}$ detailed discussion on the Ward identities in the ordinary maximal Abelian gauge can be found in [18].
}

corresponding to a residual $\mathrm{U}(1)^{n-1}$ invariance; e.g., see [19] for a nonsupersymmetric case. In contrast, in the Landau gauge, the rigid symmetry extends to the whole group $[4,12]$.

\section{The diagonal ghost equation}

Another important symmetry for the renormalization procedure is the diagonal ghost equation, given by

$$
\begin{aligned}
\mathcal{G}(S):= & \int d^{4} x d^{2} \theta\left(\frac{\delta S}{\delta c^{i}}+f^{a b i} c_{\star}^{a} \frac{\delta S}{\delta B^{b}}\right) \\
& +\int d^{4} x d^{2} \bar{\theta}\left(\frac{\delta S}{\delta \bar{c}^{i}}+f^{a b i} \bar{c}_{\star}^{a} \frac{\delta S}{\delta \bar{B}^{b}}\right) \\
& +\int d V f^{a b i} R^{a} \frac{\delta S}{\delta P^{b}} \\
= & \int d^{4} x d^{2} \theta f^{a b i} L^{a} c^{b}+\int d^{4} x d^{2} \bar{\theta} f^{a b i} \bar{L}^{a} \bar{c}^{b} \\
& -\int d V f^{a b i} \Omega^{a} V^{b} .
\end{aligned}
$$

It is important to point out here that the diagonal ghost equation can only be obtained by combining the chiral and antichiral ghosts; i.e., there is no chiral-ghost equation nor antichiral ghost equation independently. This result was already known in the case of the full Landau gauge in [20]. Also, the existence of such an identity is a signature of the Landau-type diagonal gauge-fixing condition, Eq. (6).

\section{The generalized formulation}

Noticing that the vector superfield $V$ is dimensionless, the SSMAG, given by Eqs. (5) and (6), could be written, equally well, as

$$
\begin{gathered}
\overline{\mathcal{D}}^{2} \mathcal{D}^{2}\left(V^{a}-\frac{i}{2} f^{a b i} V^{i} V^{b}+\lambda^{a A B C} V^{A} V^{B} V^{C}+O\left(V^{4}\right)\right)=0, \\
\overline{\mathcal{D}}^{2} \mathcal{D}^{2}\left(V^{i}+\eta^{i A B C} V^{A} V^{B} V^{C}+O\left(V^{4}\right)\right)=0
\end{gathered}
$$

where

$$
\begin{gathered}
\lambda^{a A B C} \in\left\{\lambda^{a b c d}, \lambda^{a b c i}, \lambda^{a b i j}, \lambda^{a i j k}\right\}, \\
\eta^{i A B C} \in\left\{\eta^{i a b c}, \eta^{i j a b}, \eta^{i j k a}, \eta^{i j k l}\right\}
\end{gathered}
$$

are invariant tensors constrained by the diagonal rigid invariance (33). In fact, these tensors are particular linear combinations of the rank-2 invariant tensors $\delta^{a b}, \delta^{i j}$ and the structure constants $f^{a b c}$ and $f^{a b i}$. The coefficients of such linear combinations are gauge parameters that might be taken to zero after the renormalization procedure, recovering then the original SSMAG. Also, it is necessary to remark that the set of Ward identities previously presented, Eqs. (24)-(34), does not prevent the redefinitions (35) and (36). 
Then, due to the above-mentioned ambiguity, it is necessary to redefine the gauge-fixing conditions (5) and (6) by the following generalized versions:

$$
\begin{aligned}
& \overline{\mathcal{D}}^{2} \mathcal{D}^{2} \omega^{a}(V)=0, \\
& \overline{\mathcal{D}}^{2} \mathcal{D}^{2} \omega^{i}(V)=0,
\end{aligned}
$$

where

$$
\begin{aligned}
\omega^{a}(V)= & V^{a}+\lambda f^{a b i} V^{i} V^{b}+\lambda^{a A B C} V^{A} V^{B} V^{C} \\
& +\lambda^{a A B C D} V^{A} V^{B} V^{C} V^{D}+\cdots, \\
\omega^{i}(V)= & V^{i}+\eta^{i A B C} V^{A} V^{B} V^{C}+\eta^{i A B C D} V^{A} V^{B} V^{C} V^{D}+\cdots .
\end{aligned}
$$

The new gauge-fixing conditions (39) and (40) are then a generalized supersymmetric version of the maximal Abelian gauge, taking into account the ambiguities arising from the absence of dimensionality of the vector superfield, and will be referred to as the GSMAG, as already mentioned in Sec. II A. According to the diagonal rigid symmetry (33), the $\lambda$ 's and $\eta$ 's tensors must obey the generalized Jacobi identities:

$$
\begin{aligned}
0= & f^{a b i} \lambda^{b c d e}+f^{c b i} \lambda^{a b d e}+f^{d b i} \lambda^{a c b e}+f^{e b i} \lambda^{a c d b} \\
0= & f^{a b i} \lambda^{b c d j}+f^{c b i} \lambda^{a b d j}+f^{d b i} \lambda^{a c b j}, \\
0= & f^{a b i} \lambda^{b c j k}+f^{c b i} \lambda^{a b j k} \\
0= & f^{a b i} \lambda^{b i j k} \\
0= & f^{a b i} \lambda^{b c d e f}+f^{c b i} \lambda^{a b d e f}+f^{d b i} \lambda^{a c b e f} \\
& +f^{e b i} \lambda^{a c d b f}+f^{f b i} \lambda^{a c d e b} \\
0= & f^{a b i} \lambda^{b c d e j}+f^{c b i} \lambda^{a b d e j}+f^{d b i} \lambda^{a c b e j}+f^{e b i} \lambda^{a c d b j} \\
0= & f^{a b i} \lambda^{b c d j k}+f^{c b i} \lambda^{a b d j k}+f^{d b i} \lambda^{a c b j k}, \\
0= & f^{a b i} \lambda^{b c j k l}+f^{c b i} \lambda^{a b j k l}, \\
0= & f^{a b i} \lambda^{b j k l m}, \\
0= & f^{a b i} \eta^{j b c d}+f^{c b i} \eta^{j a b d}+f^{d b i} \eta^{j a c b}, \\
0= & f^{a b i} \eta^{j k b c}+f^{c b i} \eta^{j k a b}, \\
0= & f^{a b i} \eta^{j k l b}, \\
0= & f^{a b i} \eta^{j b c d e}+f^{c b i} \eta^{j a b d e}+f^{d b i} \eta^{j a c b e}+f^{e b i} \eta^{j a c d b} \\
0= & f^{a b i} \eta^{j k b c d}+f^{c b i} \eta^{j k a b d}+f^{d b i} \eta^{j k a c b}, \\
0= & f^{a b i} \eta^{j k l b c}+f^{c b i} \eta^{j k l a b}, \\
0= & f^{a b i} \eta^{j k l m b},
\end{aligned}
$$

and so on. ${ }^{11}$ Thus, the gauge-fixing action (19) is replaced by

$$
\begin{aligned}
S_{\mathrm{GSMAG}}= & \frac{1}{8} s \int d V\left[c_{\star}^{a} \mathcal{D}^{2} \omega^{a}(V)+c_{\star}^{i} \mathcal{D}^{2} \omega^{i}(V)\right. \\
& \left.+\bar{c}_{\star}^{a} \overline{\mathcal{D}}^{2} \bar{\omega}^{a}(V)+\bar{c}_{\star}^{i} \overline{\mathcal{D}}^{2} \bar{\omega}^{i}(V)\right] \\
= & \frac{1}{8} \int d V\left\{B^{a} \mathcal{D}^{2} \omega^{a}(V)+B^{i} \mathcal{D}^{2} \omega^{i}(V)\right. \\
& +\bar{B}^{a} \overline{\mathcal{D}}^{2} \bar{\omega}^{a}(V)+\bar{B}^{i} \overline{\mathcal{D}}^{2} \bar{\omega}^{i}(V) \\
& -c_{\star}^{a} \mathcal{D}^{2}\left[s \omega^{a}(V)\right]-c_{\star}^{i} \mathcal{D}^{2}\left[s \omega^{i}(V)\right] \\
& \left.-\bar{c}_{\star}^{a} \overline{\mathcal{D}}^{2}\left[s \bar{\omega}^{a}(V)\right]-\bar{c}_{\star}^{i} \overline{\mathcal{D}}^{2}\left[s \bar{\omega}^{i}(V)\right]\right\},
\end{aligned}
$$

where $\bar{\omega}^{a, i}(V)$ is the complex conjugate of $\omega^{a, i}(V)$. Naturally, the external source term must be replaced by

$$
\begin{aligned}
& \Sigma_{\text {ext }}[\Omega, L, \bar{L}, R, P, \bar{R}, \bar{P}] \\
&=s\left[\int d V\left(-\Omega^{a} V^{a}-\Omega^{i} V^{i}\right)+\int d^{4} x d^{2} \theta\left(L^{a} c^{a}+L^{i} c^{i}\right)\right. \\
&+\int d^{4} x d^{2} \bar{\theta}\left(\bar{L}^{a} \bar{c}^{a}+\bar{L}^{i} \bar{c}^{i}\right)+\int d V\left(R^{a} \omega^{a}(V)\right. \\
&\left.\left.+R^{i} \omega^{i}(V)\right)+\int d V\left(\bar{R}^{a} \bar{\omega}^{a}(V)+\bar{R}^{i} \bar{\omega}^{i}(V)\right)\right] \\
&= \int d V\left[\Omega^{a}\left(s V^{a}\right)+\Omega^{i}\left(s V^{i}\right)\right] \\
&+\int d^{4} x d^{2} \theta\left[L^{a}\left(s c^{a}\right)+L^{i}\left(s c^{i}\right)\right] \\
&+\int d^{4} x d^{2} \bar{\theta}\left[\bar{L}^{a}\left(s \bar{c}^{a}\right)+\bar{L}^{i}\left(s \bar{c}^{i}\right)\right]+\int d V\left\{P^{a} \omega^{a}(V)\right. \\
&-R^{a}\left[s \omega^{a}(V)\right]+P^{i} \omega^{i}(V)-R^{i}\left[s \omega^{i}(V)\right]+\bar{P}^{a} \bar{\omega}^{a}(V) \\
&\left.-\bar{R}^{a}\left[s \bar{\omega}^{a}(V)\right]+\bar{P}^{i} \bar{\omega}^{i}(V)-\bar{R}^{i}\left[s \bar{\omega}^{i}(V)\right]\right\},
\end{aligned}
$$

where use has been made of a new set of BRST doublets of external sources

$s R^{a, i}=P^{a, i}, \quad s P^{a, i}=0, \quad s \bar{R}^{a, i}=\bar{P}^{a, i}, \quad s \bar{P}^{a, i}=0$,

with $\{\bar{R}, \bar{P}\}$ being the complex conjugate of $\{R, P\}$, respectively. The quantum numbers and the fermionic/bosonic nature of the sources (61) are those of the sources $\{R, P\}$ in Table I, even for the complex conjugate. Finally, we are in a position to replace the action (20) for a more general one:

$$
\Sigma=S_{\mathrm{SYM}}+S_{\mathrm{GSMAG}}+\Sigma_{\mathrm{ext}}[\Omega, L, \bar{L}, R, P, \bar{R}, \bar{P}] .
$$

The expression (62) above will be our starting point action, with which we will study the renormalizability. This action

\footnotetext{
${ }^{11}$ Remember here that $\{a, b, c, d, e, f\}$ are off-diagonal indices, while $\{i, j, k, l, m\}$ are diagonal ones.
} 
encodes our previous discussion about the introductory definition of the maximal Abelian gauge in superspace, originally presented in [3], and the necessity of a generalization in order to circumvent the ambiguity generated by the dimensionlessness of the vector superfield. Also, the necessary local composite operators were appropriately defined in (62). As we shall see next, a full set of Ward identities can be established for action (62). In fact, such identities are very similar to the identities (24)-(34) with few modifications.

\section{E. The Ward identities for the generalized action}

We display here a full set of Ward identities enjoyed by the action (62).

\section{The (new) Slavnov-Taylor identity}

The new Slavnov-Taylor identity now includes the BRST doublet of sources $\left\{P^{i}, R^{i}\right\},\left\{\bar{P}^{a}, \bar{R}^{a}\right\}$ and $\left\{\bar{P}^{i}, \bar{R}^{i}\right\}$ :

$$
\begin{aligned}
\mathcal{B}(\Sigma):= & \int d V\left(\frac{\delta \Sigma}{\delta \Omega^{a}} \frac{\delta \Sigma}{\delta V^{a}}+\frac{\delta \Sigma}{\delta \Omega^{i}} \frac{\delta \Sigma}{\delta V^{i}}+P^{a} \frac{\delta \Sigma}{\delta R^{a}}\right. \\
& \left.+P^{i} \frac{\delta \Sigma}{\delta R^{i}}+\bar{P}^{a} \frac{\delta \Sigma}{\delta \bar{R}^{a}}+\bar{P}^{i} \frac{\delta \Sigma}{\delta \bar{R}^{i}}\right) \\
& +\int d^{4} x d^{2} \theta\left(\frac{\delta \Sigma}{\delta L^{a}} \frac{\delta \Sigma}{\delta c^{a}}+\frac{\delta \Sigma}{\delta L^{i}} \frac{\delta \Sigma}{\delta c^{i}}+B^{a} \frac{\delta \Sigma}{\delta c_{\star}^{a}}+B^{i} \frac{\delta \Sigma}{\delta c_{\star}^{i}}\right) \\
& +\int d^{4} x d^{2} \bar{\theta}\left(\frac{\delta \Sigma}{\delta \bar{L}^{a}} \frac{\delta \Sigma}{\delta \bar{c}^{a}}+\frac{\delta \Sigma}{\delta \bar{L}^{i}} \frac{\delta \Sigma}{\delta \bar{c}^{i}}+\bar{B}^{a} \frac{\delta \Sigma}{\delta \bar{c}_{\star}^{a}}+\bar{B}^{i} \frac{\delta \Sigma}{\delta \bar{c}_{\star}^{i}}\right) \\
= & 0 .
\end{aligned}
$$

\section{The (new) diagonal gauge-fixing equations}

The new diagonal gauge-fixing equations now assume the forms:

$$
\begin{aligned}
& \frac{\delta \Sigma}{\delta B^{i}}-\frac{1}{8} \overline{\mathcal{D}}^{2} \mathcal{D}^{2} \frac{\delta \Sigma}{\delta P^{i}}=0, \\
& \frac{\delta \Sigma}{\delta \bar{B}^{i}}-\frac{1}{8} \mathcal{D}^{2} \overline{\mathcal{D}}^{2} \frac{\delta \Sigma}{\delta \bar{P}^{i}}=0 .
\end{aligned}
$$

\section{The (new) off-diagonal gauge-fixing equations}

The off-diagonal gauge-fixing equations are now very similar to the diagonal ones (we will turn to this point later):

$$
\begin{aligned}
& \frac{\delta \Sigma}{\delta B^{a}}-\frac{1}{8} \overline{\mathcal{D}}^{2} \mathcal{D}^{2} \frac{\delta \Sigma}{\delta P^{a}}=0, \\
& \frac{\delta \Sigma}{\delta \bar{B}^{a}}-\frac{1}{8} \mathcal{D}^{2} \overline{\mathcal{D}}^{2} \frac{\delta \Sigma}{\delta \bar{P}^{a}}=0 .
\end{aligned}
$$

\section{The (new) diagonal antighost equations}

The new diagonal antighost equations are modified by the presence of the sources $R^{i}$ and $\bar{R}^{i}$ :

$$
\begin{aligned}
& \frac{\delta \Sigma}{\delta c_{\star}^{i}}-\frac{1}{8} \overline{\mathcal{D}}^{2} \mathcal{D}^{2} \frac{\delta \Sigma}{\delta R^{i}}=0, \\
& \frac{\delta \Sigma}{\delta \bar{c}_{\star}^{i}}-\frac{1}{8} \mathcal{D}^{2} \overline{\mathcal{D}}^{2} \frac{\delta \Sigma}{\delta \bar{R}^{i}}=0 .
\end{aligned}
$$

\section{The (new) off-diagonal antighost equations}

The new off-diagonal antighost equations assume a simpler form in this generalized formulation:

$$
\begin{aligned}
& \frac{\delta \Sigma}{\delta c_{\star}^{a}}-\frac{1}{8} \overline{\mathcal{D}}^{2} \mathcal{D}^{2} \frac{\delta \Sigma}{\delta R^{a}}=0, \\
& \frac{\delta \Sigma}{\delta \bar{c}_{\star}^{a}}-\frac{1}{8} \mathcal{D}^{2} \overline{\mathcal{D}}^{2} \frac{\delta \Sigma}{\delta \bar{R}^{a}}=0 .
\end{aligned}
$$

\section{The (new) $\mathcal{R}$-invariance}

The new $\mathcal{R}$-invariance now includes the new sources added but it is still very similar to the previous one:

$$
\begin{aligned}
\mathcal{R}(\Sigma):= & \sum_{X \in\{V, \Omega, R, P, \bar{R}, \bar{P}\}} \int d V\left(\delta_{\mathcal{R}} X\right) \frac{\delta \Sigma}{\delta X} \\
& +\sum_{Y \in\left\{c, c_{\star}, B, L\right\}} \int d^{4} x d^{2} \theta\left(\delta_{\mathcal{R}} Y\right) \frac{\delta \Sigma}{\delta Y} \\
& +\sum_{\bar{Y} \in\left\{\bar{c}, \bar{c}_{\star}, \bar{B}, \bar{L}\right\}} \int d^{4} x d^{2} \bar{\theta}\left(\delta_{\mathcal{R}} \bar{Y}\right) \frac{\delta \Sigma}{\delta \bar{Y}} \\
= & 0,
\end{aligned}
$$

where the $\mathcal{R}$-variations are given by Eq. (32).

\section{The (new) diagonal rigid invariance}

The new diagonal rigid symmetry is also generalized as follows:

$$
\begin{aligned}
\mathcal{W}^{i}(\Sigma):= & \sum_{X \in\{V, \Omega, R, P, \bar{R}, \bar{P}\}} \int d V f^{a b i} X^{a} \frac{\delta \Sigma}{\delta X^{b}} \\
& +\sum_{Y \in\left\{c, c_{\star}, B, L\right\}} \int d^{4} x d^{2} \theta f^{a b i} Y^{a} \frac{\delta \Sigma}{\delta Y^{b}} \\
& +\sum_{\bar{Y} \in\left\{\bar{c}, \bar{c}_{\star}, \bar{B}, \bar{L}\right\}} \int d^{4} x d^{2} \bar{\theta} f^{a b i} \bar{Y}^{a} \frac{\delta \Sigma}{\delta \bar{Y}^{b}} \\
= & 0 .
\end{aligned}
$$

\section{The (new) diagonal ghost equation}

Finally, the new diagonal ghost equation is generalized in the new formulation in order to accommodate the new BRST sources. We can also notice that classical breaking term remains the same: 


$$
\begin{aligned}
\mathcal{G}(\Sigma):= & \int d^{4} x d^{2} \theta\left(\frac{\delta \Sigma}{\delta c^{i}}+f^{a b i} c_{\star}^{a} \frac{\delta S}{\delta B^{b}}\right) \\
& +\int d^{4} x d^{2} \bar{\theta}\left(\frac{\delta \Sigma}{\delta \bar{c}^{i}}+f^{a b i} \bar{c}_{\star}^{a} \frac{\delta S}{\delta \bar{B}^{b}}\right) \\
& +\int d V f^{a b i}\left(R^{a} \frac{\delta \Sigma}{\delta P^{b}}+\bar{R}^{a} \frac{\delta \Sigma}{\delta \bar{P}^{b}}\right) \\
= & \int d^{4} x d^{2} \theta f^{a b i} L^{a} c^{b}+\int d^{4} x d^{2} \bar{\theta} f^{a b i} \bar{L}^{a} \bar{c}^{b} \\
& -\int d V f^{a b i} \Omega^{a} V^{b} .
\end{aligned}
$$

The generalized Jacobi identities, Eqs. (43)-(58), and generalizations for arbitrary rank tensors are fundamental in order to establish the ghost equation as written above.

Furthermore, a deeper look at the diagonal and offdiagonal gauge-fixing equations, Eqs. (64)-(67), reveals that they are very similar and could be written in a more compact way as

$$
\begin{aligned}
& \frac{\delta \Sigma}{\delta B^{A}}-\frac{1}{8} \overline{\mathcal{D}}^{2} \mathcal{D}^{2} \frac{\delta \Sigma}{\delta P^{A}}=0, \\
& \frac{\delta \Sigma}{\delta \bar{B}^{A}}-\frac{1}{8} \mathcal{D}^{2} \overline{\mathcal{D}}^{2} \frac{\delta \Sigma}{\delta \bar{P}^{A}}=0,
\end{aligned}
$$

where $A \equiv\{a, i\}$, i.e., with no difference between the diagonal and off-diagonal sectors. It is possible because we did not give an explicit form for the field functionals (41) and (42). In fact, the split of these two sectors of the theory is provided here by the rigid symmetry (73), which is exclusively diagonal. An analogous approach was made in [4] in the context of the full Landau gauge. However, in that case, the rigid symmetry extends to the whole $\mathrm{SU}(n)$ group.

As a final comment, we would like to emphasize that the identities displayed above are, in principle, valid only at the classical level. At the quantum level it is first necessary to prove the absence of anomalies. This is in fact one of the main steps of the algebraic proof of the renormalization. The study of anomalies for such identities in superspace was exhaustively discussed in the literature; see, e.g., [10,12,21], in different gauges. In particular, as we are dealing with a pure super-Yang-Mills theory, the absence of chiral matter fields automatically guarantees the validity of the Slavnov-Taylor identity (63) and the $\mathcal{R}$-invariance (72) at a quantum level. Then, we assume from now on that the Ward identities presented here in this section are anomaly free.

\section{RENORMALIZATION}

Our next step will be to determine the most general invariant counterterm which can be freely added to all order in perturbation theory, allowing us to remove all divergences of the theory. Such a counterterm is generically written as

$$
\begin{aligned}
\Sigma_{\mathrm{CT}}= & \int d V \Delta^{(2,0,0)}(x, \theta, \bar{\theta})+\int d^{4} x d^{2} \theta \Delta^{(3,0,2)}(x, \theta) \\
& +\int d^{4} x d^{2} \bar{\theta} \Delta^{(3,0,-2)}(x, \bar{\theta}),
\end{aligned}
$$

where $\Delta^{(d, \# g, n)}$ are local polynomials in the fields and sources. The upper labels indicate the mass dimension $(d)$, the ghost number $(\# g)$, and the $\mathcal{R}$-weights $(n)$, respectively, in accordance with Table I. Also, the Hermiticity condition imposes that the antichiral polynomial $\Delta^{(3,0,-2)}$ be the chiral conjugate of the chiral polynomial $\Delta^{(3,0,2)}$.

Then, in order to find an explicit expression for the counterterm, $\Sigma_{\mathrm{CT}}$, we follow the setup of the algebraic renormalization [7] and perturb the classical action $\Sigma$, Eq. (62), by adding the counterterm described above, demanding that the perturbed action, $\left(\Sigma+\epsilon \Sigma_{\mathrm{CT}}\right)$, with $\epsilon$ being an expansion parameter, fulfill, to the first order in $\epsilon$, the same Ward identities obeyed by the classical action $\Sigma$, Eqs. (63)-(74). This amounts to imposing the following constraints on $\Sigma_{\mathrm{CT}}$ :

$$
\begin{gathered}
\mathcal{B}_{\Sigma}\left(\Sigma_{\mathrm{CT}}\right)=0, \\
\frac{\delta \Sigma_{\mathrm{CT}}}{\delta B^{i}}-\frac{1}{8} \overline{\mathcal{D}}^{2} \mathcal{D}^{2} \frac{\delta \Sigma_{\mathrm{CT}}}{\delta P^{i}}=0, \\
\frac{\delta \Sigma_{\mathrm{CT}}}{\delta \bar{B}^{i}}-\frac{1}{8} \mathcal{D}^{2} \overline{\mathcal{D}}^{2} \frac{\delta \Sigma_{\mathrm{CT}}}{\delta \bar{P}^{i}}=0, \\
\frac{\delta \Sigma_{\mathrm{CT}}}{\delta B^{a}}-\frac{1}{8} \overline{\mathcal{D}}^{2} \mathcal{D}^{2} \frac{\delta \Sigma_{\mathrm{CT}}}{\delta P^{a}}=0, \\
\frac{\delta \Sigma_{\mathrm{CT}}}{\delta \bar{B}^{a}}-\frac{1}{8} \mathcal{D}^{2} \overline{\mathcal{D}}^{2} \frac{\delta \Sigma_{\mathrm{CT}}}{\delta \bar{P}^{a}}=0, \\
\frac{\delta \Sigma_{\mathrm{CT}}}{\delta c_{\star}^{i}}-\frac{1}{8} \overline{\mathcal{D}}^{2} \mathcal{D}^{2} \frac{\delta \Sigma_{\mathrm{CT}}}{\delta R^{i}}=0, \\
\frac{\delta \Sigma_{\mathrm{CT}}}{\delta \bar{c}_{\star}^{i}}-\frac{1}{8} \mathcal{D}^{2} \overline{\mathcal{D}}^{2} \frac{\delta \Sigma_{\mathrm{CT}}}{\delta \bar{R}^{i}}=0, \\
\frac{\delta \Sigma_{\mathrm{CT}}}{\delta c_{\star}^{a}}-\frac{1}{8} \overline{\mathcal{D}}^{2} \mathcal{D}^{2} \frac{\delta \Sigma_{\mathrm{CT}}}{\delta R^{a}}=0, \\
\frac{\delta \Sigma_{\mathrm{CT}}}{\delta \bar{c}_{\star}^{a}}-\frac{1}{8} \mathcal{D}^{2} \overline{\mathcal{D}}^{2} \frac{\delta \Sigma_{\mathrm{CT}}}{\delta \bar{R}^{a}}=0, \\
\mathcal{R}\left(\Sigma_{\mathrm{CT}}\right)=0, \\
\mathcal{W}^{i}\left(\Sigma_{\mathrm{CT}}\right)=0, \\
\mathcal{G}^{i}\left(\Sigma_{\mathrm{CT}}\right)=0, \\
=0
\end{gathered}
$$

where $\mathcal{B}_{\Sigma}$ is the so-called nilpotent linearized SlavnovTaylor operator, 


$$
\begin{aligned}
\mathcal{B}_{\Sigma}= & \int d V\left(\frac{\delta \Sigma}{\delta \Omega^{a}} \frac{\delta}{\delta V^{a}}+\frac{\delta \Sigma}{\delta V^{a}} \frac{\delta}{\delta \Omega^{a}}+\frac{\delta \Sigma}{\delta \Omega^{i}} \frac{\delta}{\delta V^{i}}+\frac{\delta \Sigma}{\delta V^{i}} \frac{\delta}{\delta \Omega^{i}}\right) \\
& +\int d V\left(P^{a} \frac{\delta}{\delta R^{a}}+P^{i} \frac{\delta}{\delta R^{i}}+\bar{P}^{a} \frac{\delta}{\delta \bar{R}^{a}}+\bar{P}^{i} \frac{\delta}{\delta \bar{R}^{i}}\right) \\
& +\int d^{4} x d^{2} \theta\left(\frac{\delta \Sigma}{\delta L^{a}} \frac{\delta}{\delta c^{a}}+\frac{\delta \Sigma}{\delta c^{a}} \frac{\delta}{\delta L^{a}}+\frac{\delta \Sigma}{\delta L^{i}} \frac{\delta}{\delta c^{i}}\right. \\
& \left.+\frac{\delta \Sigma}{\delta c^{i}} \frac{\delta}{\delta L^{i}}+B^{a} \frac{\delta}{\delta c_{\star}^{a}}+B^{i} \frac{\delta}{\delta c_{\star}^{i}}\right) \\
& +\int d^{4} x d^{2} \bar{\theta}\left(\frac{\delta \Sigma}{\delta \bar{L}^{a}} \frac{\delta}{\delta \bar{c}^{a}}+\frac{\delta \Sigma}{\delta \bar{c}^{a}} \frac{\delta}{\delta \bar{L}^{a}}+\frac{\delta \Sigma}{\delta \bar{L}^{i}} \frac{\delta}{\delta \bar{c}^{i}}+\frac{\delta \Sigma}{\delta \bar{c}^{i}} \frac{\delta}{\delta \bar{L}^{i}}\right. \\
& \left.+\bar{B}^{a} \frac{\delta}{\delta \bar{c}_{\star}^{a}}+\bar{B}^{i} \frac{\delta}{\delta \bar{c}_{\star}^{i}}\right) .
\end{aligned}
$$

The presence of the nilpotent operator $\mathcal{B}_{\Sigma}$ transforms the problem of obtaining the counterterm in a cohomology problem of the operator $\mathcal{B}_{\Sigma}$. In fact, $\Sigma_{\mathrm{CT}}$ can be written as

$$
\Sigma_{\mathrm{CT}}=a_{0} S_{\mathrm{SYM}}+\mathcal{B}_{\Sigma} \Delta^{(0,-1,0)},
$$

with $a_{0}$ an arbitrary real coefficient and with $\Delta^{(0,-1,0)}$ given by

$$
\begin{aligned}
\Delta^{(0,-1,0)}= & \int d V \Delta^{(2,-1,0)}(x, \theta, \bar{\theta})+\int d^{4} x d^{2} \theta \Delta^{(3,-1,2)}(x, \theta) \\
& +\int d^{4} x d^{2} \bar{\theta} \Delta^{(3,-1,-2)}(x, \bar{\theta}),
\end{aligned}
$$

since $S_{\mathrm{SYM}}$ cannot be written as an exact $\mathcal{B}_{\Sigma}$ variation. The remaining constraints, Eqs. (79)-(89), provide that $\Delta^{(0,-1,0)}$ is written as

$$
\begin{aligned}
\Delta^{(0,-1,0)}= & \int d V\left[F^{i}(V) \Omega^{i}+F^{a}(V) \Omega^{a}\right. \\
& +G^{i}(V)\left(R^{i}+\frac{1}{8} \mathcal{D}^{2} c_{\star}^{i}\right)+G^{a}(V)\left(R^{a}+\frac{1}{8} \mathcal{D}^{2} c_{\star}^{a}\right) \\
& \left.+\bar{G}^{i}(V)\left(\bar{R}^{i}+\frac{1}{8} \overline{\mathcal{D}}^{2} \bar{c}_{\star}^{i}\right)+\bar{G}^{a}(V)\left(\bar{R}^{a}+\frac{1}{8} \overline{\mathcal{D}}^{2} \bar{c}_{\star}^{a}\right)\right] \\
& +\int d^{4} x d^{2} \theta a_{1} L^{a} c^{a}+\int d^{4} x d^{2} \bar{\theta} \bar{a}_{1} \bar{L}^{a} \bar{c}^{a},
\end{aligned}
$$

where $\left\{a_{1}, \bar{a}_{1}\right\}$ is a pair of complex conjugated arbitrary coefficients and thanks to the dimensionlessness of the vector superfield, $F^{a, i}(V)$ and $G^{a, i}(V)$ are power series in $V$ :

$$
F^{a}(V)=\alpha_{1} V^{a}+\alpha_{2} f^{a b i} V^{b} V^{i}+\sum_{n=3}^{\infty} \alpha^{a A_{1} A_{2} \ldots A_{n}} V^{A_{1}} V^{A_{2}} \ldots V^{A_{n}},
$$

$F^{i}(V)=\beta V^{i}+\sum_{n=3}^{\infty} \beta^{i A_{1} A_{2} \ldots A_{n}} V^{A_{1}} V^{A_{2}} \ldots V^{A_{n}}$,

$G^{a}(V)=\kappa_{1} V^{a}+\kappa_{2} f^{a b i} V^{b} V^{i}+\sum_{n=3}^{\infty} \kappa^{a A_{1} A_{2} \ldots A_{n}} V^{A_{1}} V^{A_{2}} \ldots V^{A_{n}}$,

$G^{i}(V)=\sigma V^{i}+\sum_{n=3}^{\infty} \sigma^{i A_{1} A_{2} \ldots A_{n}} V^{A_{1}} V^{A_{2}} \ldots V^{A_{n}}$

Naturally, $\bar{G}^{a, i}(V)$ are the complex conjugates of $G^{a, i}(V)$ and the rank- $n$ tensors: $\alpha^{a A_{1} A_{2} \ldots A_{n}}, \beta^{i A_{1} A_{2} \ldots A_{n}}, \kappa^{a A_{1} A_{2} \ldots A_{n}}$, and $\sigma^{i A_{1} A_{2} \ldots A_{n}}$ must obey generalized Jacobi identities similar to (43)-(58).

Through the action of the linearized operator $\mathcal{B}_{\Sigma}$ on $\Delta^{(0,-1,0)}$ in (93), it is possible to obtain explicit expressions for the local polynomials $\Delta^{(d, \# g, n)}$ in (77):

$$
\begin{aligned}
& \Delta^{(2,0,0)}=\left(\frac{\delta \Sigma}{\delta \Omega^{j}} \frac{\partial F^{i}(V)}{\partial V^{j}}+\frac{\delta \Sigma}{\delta \Omega^{a}} \frac{\partial F^{i}(V)}{\partial V^{a}}\right) \Omega^{i}+F^{i}(V) \frac{\delta \Sigma}{\delta V^{i}}+\left(\frac{\delta \Sigma}{\delta \Omega^{i}} \frac{\partial F^{a}(V)}{\partial V^{i}}+\frac{\delta \Sigma}{\delta \Omega^{b}} \frac{\partial F^{a}(V)}{\partial V^{b}}\right) \Omega^{a}+F^{a}(V) \frac{\delta \Sigma}{\delta V^{a}} \\
&+\left(\frac{\delta \Sigma}{\delta \Omega^{j}} \frac{\partial G^{i}(V)}{\partial V^{j}}+\frac{\delta \Sigma}{\delta \Omega^{a}} \frac{\partial G^{i}(V)}{\partial V^{a}}\right)\left(R^{i}+\frac{1}{8} \mathcal{D}^{2} c_{\star}^{i}\right)+G^{i}(V) P^{i}+\frac{1}{8} B^{i} \mathcal{D}^{2} G^{i}(V) \\
&+\left(\frac{\delta \Sigma}{\delta \Omega^{i}} \frac{\partial G^{a}(V)}{\partial V^{i}}+\frac{\delta \Sigma}{\delta \Omega^{b}} \frac{\partial G^{a}(V)}{\partial V^{b}}\right)\left(R^{a}+\frac{1}{8} \mathcal{D}^{2} c_{\star}^{a}\right)+G^{a}(V) P^{a}+\frac{1}{8} B^{a} \mathcal{D}^{2} G^{a}(V) \\
&+\left(\frac{\delta \Sigma}{\delta \Omega^{j}} \frac{\partial \bar{G}^{i}(V)}{\partial V^{j}}+\frac{\delta \Sigma}{\delta \Omega^{a}} \frac{\partial \bar{G}^{i}(V)}{\partial V^{a}}\right)\left(\bar{R}^{i}+\frac{1}{8} \overline{\mathcal{D}}^{2} \bar{c}_{\star}^{i}\right)+\bar{G}^{i}(V) \bar{P}^{i}+\frac{1}{8} \bar{B}^{i} \overline{\mathcal{D}}^{2} \bar{G}^{i}(V) \\
&+\left(\frac{\delta \Sigma}{\delta \Omega^{i}} \frac{\partial \bar{G}^{a}(V)}{\partial V^{i}}+\frac{\delta \Sigma}{\delta \Omega^{b}} \frac{\partial \bar{G}^{a}(V)}{\partial V^{b}}\right)\left(\bar{R}^{a}+\frac{1}{8} \overline{\mathcal{D}}^{2} \bar{c}_{\star}^{a}\right)+\bar{G}^{a}(V) \bar{P}^{a}+\frac{1}{8} \bar{B}^{a} \overline{\mathcal{D}}^{2} \bar{G}^{a}(V), \\
& \Delta^{(3,0,2)}=-\frac{a_{0}}{128 g^{2}}\left(W^{a \alpha} W_{\alpha}^{a}+W^{i \alpha} W_{\alpha}^{i}\right)+a_{1} \frac{\delta \Sigma}{\delta c^{a}} c^{a}+a_{1} L^{a} \frac{\delta \Sigma}{\delta L^{a}},
\end{aligned}
$$




$$
\Delta^{(3,0,-2)}=-\frac{a_{0}}{128 g^{2}}\left(\bar{W}_{\dot{\alpha}}^{a} \bar{W}^{a \dot{\alpha}}+\bar{W}_{\dot{\alpha}}^{i} \bar{W}^{i \dot{\alpha}}\right)+\bar{a}_{1} \frac{\delta \Sigma}{\delta \bar{c}^{a}} \bar{c}^{a}+\bar{a}_{1} \bar{L}^{a} \frac{\delta \Sigma}{\delta \bar{L}^{a}} .
$$

Furthermore, it is useful to write the counterterm in a parametric form. Then, in order to establish such an expression, let us first notice that the nontrivial part of the counterterm can be easily rewritten as

$$
a_{0} S_{\mathrm{SYM}}=-\frac{a_{0}}{128 g^{2}} \int d^{4} x d^{2} \theta W^{A \alpha} W_{\alpha}^{A}+\text { c.c. }=-a_{0} g^{2} \frac{\partial S_{\mathrm{SYM}}}{\partial g^{2}}=-a_{0} g^{2} \frac{\partial \Sigma}{\partial g^{2}},
$$

while the remaining terms of (91), i.e., the so-called trivial part of the cohomology, are written in the same fashion. Namely,

$$
\begin{aligned}
& \mathcal{B}_{\Sigma} \Delta^{(0,-1,0)}=\int d V\left(F^{a}(V) \frac{\delta \Sigma}{\delta V^{a}}+\kappa_{1} R^{a} \frac{\delta \Sigma}{\delta R^{a}}+\kappa_{1} P^{a} \frac{\delta \Sigma}{\delta P^{a}}+\bar{\kappa}_{1} \bar{R}^{a} \frac{\delta \Sigma}{\delta \bar{R}^{a}}+\bar{\kappa}_{1} \bar{P}^{a} \frac{\delta \Sigma}{\delta \bar{P}^{a}}\right)-\int d V\left(\Omega^{b} \frac{\partial F^{b}(V)}{\partial V^{a}}+\Omega^{i} \frac{\partial F^{i}(V)}{\partial V^{a}}\right) \frac{\delta \Sigma}{\delta \Omega^{a}} \\
& +\int d V\left(F^{i}(V) \frac{\delta \Sigma}{\delta V^{i}}+\sigma R^{i} \frac{\delta \Sigma}{\delta R^{i}}+\sigma P^{i} \frac{\delta \Sigma}{\delta P^{i}}+\bar{\sigma} \bar{R}^{i} \frac{\delta \Sigma}{\delta \bar{R}^{i}}+\bar{\sigma} \bar{P}^{i} \frac{\delta \Sigma}{\delta \bar{P}^{i}}\right)-\int d V\left(\Omega^{a} \frac{\partial F^{a}(V)}{\partial V^{i}}+\Omega^{j} \frac{\partial F^{j}(V)}{\partial V^{i}}\right) \frac{\delta \Sigma}{\delta \Omega^{i}} \\
& +\int d^{4} x d^{2} \theta\left(a_{1} L^{a} \frac{\delta \Sigma}{\delta L^{a}}-a_{1} c^{a} \frac{\delta \Sigma}{\delta c^{a}}+\kappa_{1} c_{\star}^{a} \frac{\delta \Sigma}{\delta c_{\star}^{a}}+\kappa_{1} B^{a} \frac{\delta \Sigma}{\delta B^{a}}+\sigma c_{\star}^{i} \frac{\delta \Sigma}{\delta c_{\star}^{i}}+\sigma B^{i} \frac{\delta \Sigma}{\delta B^{i}}\right) \\
& +\int d^{4} x d^{2} \bar{\theta}\left(\bar{a}_{1} \bar{L}^{a} \frac{\delta \Sigma}{\delta \bar{L}^{a}}-\bar{a}_{1} \bar{c}^{a} \frac{\delta \Sigma}{\delta \bar{c}^{a}}+\bar{\kappa}_{1} \bar{c}_{\star}^{a} \frac{\delta \Sigma}{\delta \bar{c}_{\star}^{a}}+\bar{\kappa}_{1} \bar{B}^{a} \frac{\delta \Sigma}{\delta \bar{B}^{a}}+\bar{\sigma} \bar{c}_{\star}^{i} \frac{\delta \Sigma}{\delta \bar{c}_{\star}^{i}}+\bar{\sigma} \bar{B}^{i} \frac{\delta \Sigma}{\delta \bar{B}^{i}}\right) \\
& +\left(\kappa_{2}-\kappa_{1} \lambda\right) \frac{\partial \Sigma}{\partial \lambda}+\sum_{n=3}^{\infty}\left(\kappa^{a A_{1} A_{2} \ldots A_{n}}-\kappa_{1} \lambda^{a A_{1} A_{2} \ldots A_{n}}\right) \frac{\partial \Sigma}{\partial \lambda^{a A_{1} A_{2} \ldots A_{n}}} \\
& +\left(\bar{\kappa}_{2}-\bar{\kappa}_{1} \bar{\lambda}\right) \frac{\partial \Sigma}{\partial \bar{\lambda}}+\sum_{n=3}^{\infty}\left(\bar{\kappa}^{a A_{1} A_{2} \ldots A_{n}}-\bar{\kappa}_{1} \bar{\lambda}^{a A_{1} A_{2} \ldots A_{n}}\right) \frac{\partial \Sigma}{\partial \bar{\lambda}^{a A_{1} A_{2} \ldots A_{n}}} \\
& +\sum_{n=3}^{\infty}\left(\sigma^{i A_{1} A_{2} \ldots A_{n}}-\sigma \eta^{i A_{1} A_{2} \ldots A_{n}}\right) \frac{\partial \Sigma}{\partial \eta^{i A_{1} A_{2} \ldots A_{n}}}+\sum_{n=3}^{\infty}\left(\bar{\sigma}^{i A_{1} A_{2} \ldots A_{n}}-\bar{\sigma} \bar{\eta}^{i A_{1} A_{2} \ldots A_{n}}\right) \frac{\partial \Sigma}{\partial \bar{\eta}^{i A_{1} A_{2} \ldots A_{n}}} .
\end{aligned}
$$

Combining (101) and (102), the counterterm can be viewed as

$$
\Sigma_{\mathrm{CT}}=\mathcal{O} \Sigma,
$$

where $\mathcal{O}$ is a linear operator acting on $\Sigma$ being given by

$$
\begin{aligned}
& \mathcal{O}=-a_{0} g^{2} \frac{\partial}{\partial g^{2}}+\int d V\left(F^{a}(V) \frac{\delta}{\delta V^{a}}+\kappa_{1} R^{a} \frac{\delta}{\delta R^{a}}+\kappa_{1} P^{a} \frac{\delta}{\delta P^{a}}+\bar{\kappa}_{1} \bar{R}^{a} \frac{\delta}{\delta \bar{R}^{a}}+\bar{\kappa}_{1} \bar{P}^{a} \frac{\delta}{\delta \bar{P}^{a}}\right)-\int d V\left(\Omega^{b} \frac{\partial F^{b}(V)}{\partial V^{a}}+\Omega^{i} \frac{\partial F^{i}(V)}{\partial V^{a}}\right) \frac{\delta}{\delta \Omega^{a}} \\
& +\int d V\left(F^{i}(V) \frac{\delta}{\delta V^{i}}+\sigma R^{i} \frac{\delta}{\delta R^{i}}+\sigma P^{i} \frac{\delta}{\delta P^{i}}+\bar{\sigma} \bar{R}^{i} \frac{\delta}{\delta \bar{R}^{i}}+\bar{\sigma} \bar{P}^{i} \frac{\delta}{\delta \bar{P}^{i}}\right)-\int d V\left(\Omega^{a} \frac{\partial F^{a}(V)}{\partial V^{i}}+\Omega^{j} \frac{\partial F^{j}(V)}{\partial V^{i}}\right) \frac{\delta}{\delta \Omega^{i}} \\
& +\int d^{4} x d^{2} \theta\left(a_{1} L^{a} \frac{\delta}{\delta L^{a}}-a_{1} c^{a} \frac{\delta}{\delta c^{a}}+\kappa_{1} c_{\star}^{a} \frac{\delta}{\delta c_{\star}^{a}}+\kappa_{1} B^{a} \frac{\delta}{\delta B^{a}}+\sigma c_{\star}^{i} \frac{\delta}{\delta c_{\star}^{i}}+\sigma B^{i} \frac{\delta}{\delta B^{i}}\right) \\
& +\int d^{4} x d^{2} \bar{\theta}\left(\bar{a}_{1} \bar{L}^{a} \frac{\delta}{\delta \bar{L}^{a}}-\bar{a}_{1} \bar{c}^{a} \frac{\delta}{\delta \bar{c}^{a}}+\bar{\kappa}_{1} \bar{c}_{\star}^{a} \frac{\delta}{\delta \bar{c}_{\star}^{a}}+\bar{\kappa}_{1} \bar{B}^{a} \frac{\delta}{\delta \bar{B}^{a}}+\bar{\sigma} \bar{c}_{\star}^{i} \frac{\delta}{\delta \bar{c}_{\star}^{i}}+\bar{\sigma} \bar{B}^{i} \frac{\delta}{\delta \bar{B}^{i}}\right) \\
& +\left(\kappa_{2}-\kappa_{1} \lambda\right) \frac{\partial}{\partial \lambda}+\sum_{n=3}^{\infty}\left(\kappa^{a A_{1} A_{2} \ldots A_{n}}-\kappa_{1} \lambda^{a A_{1} A_{2} \ldots A_{n}}\right) \frac{\partial}{\partial \lambda^{a A_{1} A_{2} \ldots A_{n}}}+\left(\bar{\kappa}_{2}-\bar{\kappa}_{1} \bar{\lambda}\right) \frac{\partial}{\partial \bar{\lambda}}+\sum_{n=3}^{\infty}\left(\bar{\kappa}^{a A_{1} A_{2} \ldots A_{n}}-\bar{\kappa}_{1} \bar{\lambda}^{a A_{1} A_{2} \ldots A_{n}}\right) \frac{\partial}{\partial \bar{\lambda}^{a A_{1} A_{2} \ldots A_{n}}} \\
& +\sum_{n=3}^{\infty}\left(\sigma^{i A_{1} A_{2} \ldots A_{n}}-\sigma \eta^{i A_{1} A_{2} \ldots A_{n}}\right) \frac{\partial}{\partial \eta^{i A_{1} A_{2} \ldots A_{n}}}+\sum_{n=3}^{\infty}\left(\bar{\sigma}^{i A_{1} A_{2} \ldots A_{n}}-\bar{\sigma} \bar{\eta}^{i A_{1} A_{2} \ldots A_{n}}\right) \frac{\partial}{\partial \bar{\eta}^{i A_{1} A_{2} \ldots A_{n}}} .
\end{aligned}
$$

Now, in order to find the renormalization of the fields, sources, and parameters of the theory, let us first define a variable $\Phi$ representing all these quantities, i.e.,

$$
\Phi \equiv V, B, \bar{B}, c, \bar{c}, c_{\star}, \bar{c}_{\star}, \Omega, L, \bar{L}, R, \bar{R}, P, \bar{P}, g, \lambda, \bar{\lambda}, \lambda^{a A_{1} A_{2} \cdots}, \bar{\lambda}^{a A_{1} A_{2} \cdots}, \eta^{i A_{1} A_{2} \cdots}, \bar{\eta}^{a A_{1} A_{2} \cdots} .
$$


Thus, the bare quantities $\Phi_{0}$ are related to the renormalized ones by

$$
\Phi_{0}=\Phi+\epsilon \zeta_{\Phi}+O\left(\epsilon^{2}\right),
$$

where $\zeta_{\Phi}$ is assumed to have a general form of a local functional of the fields. This assumption is necessary in view of the possibility of nonlinear and/or matricial renormalizations. ${ }^{12}$ Then, the bare classical action $\Sigma\left[\Phi_{0}\right]$ is related to the renormalized action $\Sigma[\Phi]$ as

$\Sigma\left[\Phi_{0}\right]=\Sigma\left[\Phi+\epsilon \zeta_{\Phi}\right]=\Sigma[\Phi]+\epsilon \zeta_{\Phi} \frac{\mathrm{d} \Sigma}{\mathrm{d} \Phi}+O\left(\epsilon^{2}\right)$,

where the derivative $d / d \Phi$ is a partial derivative when $\Phi$ represents a parameter and an integrated functional derivative when $\Phi$ represents a field or a source. Now, the expression above can be compared with

$$
\Sigma\left[\Phi_{0}\right]=\Sigma[\Phi]+\epsilon \Sigma_{\mathrm{CT}}+O\left(\epsilon^{2}\right)=\Sigma[\Phi]+\epsilon \mathcal{O} \Sigma+O\left(\epsilon^{2}\right),
$$

which comes from perturbation theory and from (103). Therefore,

$$
\mathcal{O} \equiv \zeta_{\Phi} \frac{\mathrm{d}}{\mathrm{d} \Phi}
$$

Then, the counterterm can be reabsorbed in the starting point action by the following renormalizations:

$$
\Phi_{0}=\Phi+\epsilon \mathcal{O} \Phi+O\left(\epsilon^{2}\right) .
$$

Taking the last expression into account, the components of the vector superfield renormalize, up to order $\epsilon$, as

$$
\begin{aligned}
& V_{0}^{a}=V^{a}+\epsilon F^{a}(V), \\
& V_{0}^{i}=V^{i}+\epsilon F^{i}(V),
\end{aligned}
$$

while the sources $\Omega^{a, i}$ renormalize as

$$
\begin{aligned}
& \Omega_{0}^{a}=\Omega^{a}-\epsilon\left(\Omega^{b} \frac{\partial F^{b}(V)}{\partial V^{a}}+\Omega^{i} \frac{\partial F^{i}(V)}{\partial V^{a}}\right), \\
& \Omega_{0}^{i}=\Omega^{i}-\epsilon\left(\Omega^{a} \frac{\partial F^{a}(V)}{\partial V^{i}}+\Omega^{j} \frac{\partial F^{j}(V)}{\partial V^{i}}\right) .
\end{aligned}
$$

The remaining fields and sources renormalize as

$$
\begin{gathered}
c_{0}^{a}=Z c^{a}, \\
L_{0}^{a}=Z^{-1} L^{a}, \\
\bar{c}_{0}^{a}=\bar{Z} \bar{c}^{a},
\end{gathered}
$$

\footnotetext{
${ }^{12}$ In its simplest form, $\zeta_{\Phi}$ is proportional to its corresponding $\Phi$ times a constant.
}

$$
\begin{gathered}
\bar{L}_{0}^{a}=\bar{Z}^{-1} \bar{L}^{a}, \\
\left(c_{\star}^{a}, B^{a}, R^{a}, P^{a}\right)_{0}=Z_{\star}\left(c_{\star}^{a}, B^{a}, R^{a}, P^{a}\right), \\
\left(\bar{c}_{\star}^{a}, \bar{B}^{a}, \bar{R}^{a}, \bar{P}^{a}\right)_{0}=\bar{Z}_{\star}\left(\bar{c}_{\star}^{a}, \bar{B}^{a}, \bar{R}^{a}, \bar{P}^{a}\right), \\
\left(c_{\star}^{i}, B^{i}, R^{i}, P^{i}\right)_{0}=\mathcal{Z}_{\star}\left(c_{\star}^{i}, B^{i}, R^{i}, P^{i}\right), \\
\left(\bar{c}_{\star}^{i}, \bar{B}^{i}, \bar{R}^{i}, \bar{P}^{i}\right)_{0}=\overline{\mathcal{Z}}_{\star}\left(\bar{c}_{\star}^{i}, \bar{B}^{i}, \bar{R}^{i}, \bar{P}^{i}\right), \\
\left(c^{i}, \bar{c}^{i}, L^{i}, \bar{L}^{i}\right)_{0}=\left(c^{i}, \bar{c}^{i}, L^{i}, \bar{L}^{i}\right),
\end{gathered}
$$

with

$$
\begin{gathered}
Z=1-\epsilon a_{1}, \\
Z^{-1}=1+\epsilon a_{1}, \\
\bar{Z}=1-\epsilon \bar{a}_{1}, \\
\bar{Z}^{-1}=1+\epsilon \bar{a}_{1}, \\
Z_{\star}=1+\epsilon \kappa_{1}, \\
\bar{Z}_{\star}=1+\epsilon \bar{\kappa}_{1}, \\
\mathcal{Z}_{\star}=1+\epsilon \sigma, \\
\overline{\mathcal{Z}}_{\star}=1+\epsilon \bar{\sigma} .
\end{gathered}
$$

Finally, the parameters renormalize as

$$
\begin{gathered}
g_{0}=Z_{g} g=\left(1-\epsilon a_{0}\right) g, \\
\lambda_{0}=\lambda+\epsilon\left(\kappa_{2}-\kappa_{1} \lambda\right), \\
\lambda_{0}^{a A_{1} A_{2} \cdots}=\lambda^{a A_{1} A_{2} \cdots}+\epsilon\left(\kappa^{a A_{1} A_{2} \cdots}-\kappa_{1} \lambda^{a A_{1} A_{2} \cdots}\right), \\
\bar{\lambda}_{0}^{a A_{1} A_{2} \cdots}=\bar{\lambda}^{a A_{1} A_{2} \cdots}+\epsilon\left(\bar{\kappa}^{a A_{1} A_{2} \cdots}-\bar{\kappa}_{1} \bar{\lambda}^{a A_{1} A_{2} \cdots}\right), \\
\eta_{0}^{a A_{1} A_{2} \cdots}=\eta^{a A_{1} A_{2} \cdots}+\epsilon\left(\sigma^{a A_{1} A_{2} \cdots}-\sigma \eta^{a A_{1} A_{2} \cdots}\right), \\
\bar{\eta}_{0}^{a A_{1} A_{2} \cdots}=\bar{\eta}^{a A_{1} A_{2} \cdots}+\epsilon\left(\bar{\sigma}^{a A_{1} A_{2} \cdots}-\bar{\sigma} \bar{\eta}^{a A_{1} A_{2} \cdots}\right) .
\end{gathered}
$$

These expressions end the proof of the renormalizability of the theory, but some comments about the expressions above are necessary. First, notice that the renormalizations of the components of the vector superfield are nonlinear as $F^{a, i}(V)$ are power series in $V$, as stated in Eqs. (94) and (95). It is also clear from Eqs. (94) and (95) that the diagonal and off-diagonal components of $V$ are mixed. In other words, the renormalization between $V^{a}$ and $V^{i}$ is matricial. It can be put in a clear way by noticing that the power series $F^{a, i}(V)$ can always be written as

$$
\begin{gathered}
F^{a}(V)=\alpha_{1} V^{a}+F^{a b}(V) V^{b}+F^{a i}(V) V^{i}, \\
F^{i}(V)=\beta V^{i}+F^{i a}(V) V^{a}+F^{i j}(V) V^{j},
\end{gathered}
$$

with $F^{a i}(V) \neq F^{i a}(V)$ in general. Then we have the following matricial renormalization: 


$$
\left(\begin{array}{c}
V_{0}^{a} \\
V_{0}^{i}
\end{array}\right)=\left(\begin{array}{ll}
Z_{V}^{a b} & Z_{V}^{a j} \\
Z_{V}^{i b} & Z_{V}^{i j}
\end{array}\right)\left(\begin{array}{l}
V^{b} \\
V^{j}
\end{array}\right),
$$

where

$$
\begin{gathered}
Z_{V}^{a b}=\delta^{a b}+\epsilon\left[\alpha_{1} \delta^{a b}+F^{a b}(V)\right], \\
Z_{V}^{a j}=\epsilon F^{a j}(V), \\
Z_{V}^{i b}=\epsilon F^{i b}(V), \\
Z_{V}^{i j}=\delta^{i j}+\epsilon\left[\beta \delta^{i j}+F^{i j}(V)\right] .
\end{gathered}
$$

The expressions (113) and (114) for the renormalizations of the sources $\Omega^{a, i}$ also indicate matricial and nonlinear renormalizations:

$$
\left(\begin{array}{l}
\Omega_{0}^{a} \\
\Omega_{0}^{i}
\end{array}\right)=\left(\begin{array}{cc}
Z_{\Omega}^{a b} & Z_{\Omega}^{a j} \\
Z_{\Omega}^{i b} & Z_{\Omega}^{i j}
\end{array}\right)\left(\begin{array}{l}
\Omega^{b} \\
\Omega^{j}
\end{array}\right),
$$

where

$$
\begin{gathered}
Z_{\Omega}^{a b}=\delta^{a b}-\epsilon \frac{\partial F^{b}(V)}{\partial V^{a}}, \\
Z_{\Omega}^{a j}=-\epsilon \frac{\partial F^{j}(V)}{\partial V^{a}}, \\
Z_{\Omega}^{i b}=-\epsilon \frac{\partial F^{b}(V)}{\partial V^{i}}, \\
Z_{\Omega}^{i j}=\delta^{i j}-\epsilon \frac{\partial F^{j}(V)}{\partial V^{i}} .
\end{gathered}
$$

After the removal of the divergences of the theory, the original SSMAG can now be reobtained by choosing values for the gauge parameters. In fact, the particular choices

$$
\begin{aligned}
\lambda & =-\frac{i}{2}, \quad \bar{\lambda}=+\frac{i}{2}, \\
\lambda^{a A_{1} A_{2} \ldots} & =\bar{\lambda}^{a A_{1} A_{2} \ldots}=\eta^{i A_{1} A_{2} \cdots}=\bar{\eta}^{i A_{1} A_{2} \cdots}=0,
\end{aligned}
$$

lead us from the GSMAG to the SSMAG.

A final comment emerges from a comparison between the supersymmetric and ordinary cases. In the study of the renormalization of the Yang-Mills action quantized in the maximal Abelian gauge, the absence of the off-diagonal gauge-fixing and antighost equations as genuine Ward identities gives rise to extra interaction terms among the ghost fields. In fact, quartic interaction ghost terms naturally emerge, as a diagrammatic analysis reveals, and the original gauge can only be defined modulo an extra gauge parameter [19]. In the earlier paper [3], when the SSMAG was first presented, we also proposed possible quartic interaction ghost terms following the nonsupersymmetric approach. However, on that occasion we did not realize that the off-diagonal gauge-fixing and antighost equations, given by Eqs. (26), (27), (29), and (30) for the SSMAG, could be established in the supersymmetric scenario, nor that the general approach, given by the GSMAG, should be implemented.

\section{CONCLUSIONS AND PERSPECTIVES}

In this work, we have concluded the algebraic proof of the renormalizability of a $N=1$ super-Yang-Mills theory for the $\mathrm{SU}(n)$ group in a supersymmetric version of the maximal Abelian gauge. A generalized version of the original proposal, Ref. [3], has been adopted. We call this extended version the generalized supermaximal Abelian gauge). Such a version depends on a set of infinity gauge parameters but, at the end, the original version, called the simplest supermaximal Abelian gauge), can be achieved from the generalized one by a suitable adjusting of the gauge parameters, which are, however, fundamental in the algebraic proof.

The proof presented here is very similar to the one presented in [4] in the case of the Landau gauge. The main difference is that the gauge symmetry group is explicitly split into its diagonal and off-diagonal parts. This split is made evident from the diagonal rigid symmetry (73) and the consequent generalized Jacobi identities (43)-(58) enjoyed by the invariant tensors $\lambda$ and $\eta$ (the corresponding gauge parameters are "hidden" in these tensors).

Also, in [4] a gauge invariant mass term is introduced. This invariant mass term is constructed by means of a gauge invariant composite superfield, $\mathbf{V}(V, \Xi, \bar{\Xi})$, given by

$$
\exp [\mathbf{V}(V, \Xi, \bar{\Xi})]=e^{-i \overline{\bar{\Xi}}} e^{V} e^{i \Xi},
$$

where $V=V^{A} T^{A}$ is the usual vector superfield and $\{\Xi, \bar{\Xi}\}$ the pair of chiral conjugated Stueckelberg-like superfields. Being dimensionless, the invariant composite field $\mathbf{V}$ gives rise to the following mass term:

$$
\begin{aligned}
S_{m^{2}}= & m^{2} \int d V\left(\mathbf{V}^{A} \mathbf{V}^{A}+t^{A B C D} \mathbf{V}^{A} \mathbf{V}^{B} \mathbf{V}^{C} \mathbf{V}^{D}\right. \\
& \left.+t^{A B C D E} \mathbf{V}^{A} \mathbf{V}^{B} \mathbf{V}^{C} \mathbf{V}^{D} \mathbf{V}^{E}+\cdots\right),
\end{aligned}
$$

where $m^{2}$ is a mass squared parameter and $t^{A B C D \ldots}$ are invariant tensors. It can be immediately observed that there is a mass degeneracy among the $\left(n^{2}-1\right)$ directions of the group. Therefore, once we have at our disposal the GSMAG, which naturally splits the diagonal and offdiagonal sectors of the group, it is possible to partially break the mass degeneracy and define two different mass parameters, one for the $(n-1)$ diagonal components and the other one for the $n(n-1)$ off-diagonal components. This approach is already being developed in the context of the ordinary Yang-Mills [22], opening a way for the study of the so-called Abelian dominance conjecture.

Another problem that can be investigated in the GSMAG is the Gribov problem $[23,24]$. In the Landau gauge this problem was first investigated in superspace in Ref. [25]. In the nonsupersymmetric scenario, the Gribov ambiguity 
problem was extensively investigated in the maximal Abelian gauge in Refs. [26-30].

\section{ACKNOWLEDGMENTS}

The Conselho Nacional de Desenvolvimento Científico e Tecnológico (CNPq-Brazil), the Coordenação de Aperfeiçoamento de Pessoal de Nível Superior (CAPES), and the SR2-UERJ are gratefully acknowledged. M. A. L. Capri is a level PQ-2 researcher under the program Produtividade em Pesquisa-CNPq, Grant No. 307783/ 2014-6 and is a Procientista under SR2-UERJ. R. C. T. is supported by the Coordenação de Aperfeiçoamento de Pessoal de Nível Superior (CAPES) under the program Doutorado Sanduíche no Exterior (PDSE), Grant No. 88881.188419/2018-01.

\section{APPENDIX: THE MAXIMAL ABELIAN GAUGE FOR ORDINARY SU $(n)$ YANG-MILLS THEORY}

In this section, we make a brief review on the ordinary maximal Abelian gauge. This gauge arises from the breaking of color symmetry, which generates a separation of the structure of the group $\mathrm{SU}(n)$. It is well known that the gauge field is defined in the adjoint representation of the $\mathrm{SU}(n)$ group, namely,

$$
A_{\mu}(x)=A_{\mu}^{A}(x) T^{A},
$$

where the index $A$ runs from 1 to $\left(n^{2}-1\right)$ and $T^{A}$ stands for a set of Hermitian traceless matrices forming the generators of the group. These generators can be split into a diagonal sector and an off-diagonal sector, $T^{A} \equiv\left\{T^{a}, T^{i}\right\}$, where the indices $\{a, b, c, \ldots\}=1, \ldots, n(n-1)$ are the so-called offdiagonal indices, connected to the non-Abelian sector, while the indices $\{i, j, k, \ldots\}=1, \ldots, n-1$ are the diagonal ones, related to the Abelian subgroup of $\mathrm{SU}(n)$. In this way, the Lie algebra,

$$
\left[T^{A}, T^{B}\right]=i f^{A B C} T^{C},
$$

is rewritten in terms of the diagonal and off-diagonal components, i.e.,

$$
\begin{gathered}
{\left[T^{a}, T^{b}\right]=i f^{a b c} T^{c}+i f^{a b i} T^{i},} \\
{\left[T^{a}, T^{i}\right]=-i f^{a b i} T^{c},} \\
{\left[T^{i}, T^{j}\right]=0,}
\end{gathered}
$$

where $f^{a b c}$ and $f^{a b i}$ are the structure constants of the group. These constants obey the following Jacobi's relations:

$$
\begin{gathered}
0=f^{a b i} f^{b c j}+f^{a b j} f^{b i c}, \\
0=f^{a b c} f^{c d i}+f^{a d c} f^{c i b}+f^{a i c} f^{c b d}, \\
0=f^{a b c} f^{c d e}+f^{a b i} f^{i d e}+f^{a d c} f^{c e d}+f^{a b i} f^{i e b} \\
+f^{a e c} f^{c b d}+f^{a e i} f^{i b d},
\end{gathered}
$$

which are derived from

$$
f^{A B C} f^{C D E}+f^{A D C} f^{C E B}+f^{A E C} f^{C B D}=0 .
$$

Now the gauge field can be split in terms of the two components of the group,

$$
A_{\mu}(x)=A_{\mu}^{A}(x) T^{A}=A_{\mu}^{a}(x) T^{a}+A_{\mu}^{i}(x) T^{i} .
$$

Then, the Yang-Mills action can also be written in terms of its Abelian and non-Abelian components,

$$
\begin{aligned}
S_{\mathrm{YM}} & =-\frac{1}{2 g^{2}} \operatorname{Tr} \int d^{4} x F^{\mu \nu} F_{\mu \nu} \\
& =-\frac{1}{4 g^{2}} \int d^{4} x\left(F^{a \mu \nu} F_{\mu \nu}^{a}+F^{i \mu \nu} F_{\mu \nu}^{i}\right),
\end{aligned}
$$

where

$$
\operatorname{Tr}\left(T^{A} T^{B}\right)=\frac{\delta^{A B}}{2}
$$

and

$$
\begin{gathered}
F_{\mu \nu}=F^{A} T^{A}=F_{\mu \nu}^{a} T^{a}+F_{\mu \nu}^{i} T^{i}, \\
F_{\mu \nu}^{a}=D_{\mu}^{a b} A_{\nu}^{b}-D_{\nu}^{a b} A_{\mu}^{b}+f^{a b c} A_{\mu}^{a} A_{\nu}^{b}, \\
F_{\mu \nu}^{i}=\partial_{\mu} A_{\nu}^{i}-\partial_{\nu} A_{\mu}^{i}+f^{a b i} A_{\mu}^{a} A_{\nu}^{b}, \\
D_{\mu}^{a b}=\delta^{a b} \partial_{\mu}-f^{a b i} A_{\mu}^{i} .
\end{gathered}
$$

Moreover, this action is invariant by the following infinitesimal transformations,

$$
\begin{gathered}
A_{\mu}^{i} \rightarrow\left(A^{\omega}\right)_{\mu}^{i}=A_{\mu}^{i}-\left(\partial_{\mu} \omega^{i}+f^{a b i} A_{\mu}^{a} \omega^{b}\right), \\
A_{\mu}^{a} \rightarrow\left(A^{\omega}\right)_{\mu}^{a}=A_{\mu}^{a}-\left(D_{\mu}^{a b} \omega^{b}+f^{a b c} A_{\mu}^{b} \omega^{c}+f^{a b i} A_{\mu}^{b} \omega^{i}\right),
\end{gathered}
$$

where the infinitesimal gauge parameter, $\omega$, can also be split in terms of the two sectors of the group:

$$
\omega(x)=\omega^{i}(x) T^{i}+\omega^{a}(x) T^{a} .
$$

Since the Yang-Mills action can be divided from the point of view of the group structure, let us now verify which are the consequences arising from the quantization process of this theory. Let us start by defining the generator functional for the Green functions of the theory,

$$
Z[J] \propto \int[d A] \exp \left(i S_{\mathrm{YM}}+i \int d^{4} x J^{A \mu} A_{\mu}^{A}\right) .
$$

However, since gauge symmetry is preserved, the functional integration measure, $[d A]$, overcounts equivalent configurations in the field space and therefore an additional condition, or constraint, must be imposed so that spurious degrees of freedom are eliminated. This constraint is the so-called "gauge-fixing condition" and, according to the Faddeev-Popov quantization approach [16], it is introduced in the functional integrals as follows: 


$$
\begin{aligned}
Z[J] \propto & \int[d A] \delta(G) \operatorname{det}\left|\frac{\delta G\left[A^{\omega}\right]}{\delta \omega}\right| \\
& \times \exp \left(i S_{\mathrm{YM}}+i \int d^{4} x J^{A \mu} A_{\mu}^{A}\right) .
\end{aligned}
$$

Here, the $G$ functional plays the role of setting the necessary conditions to correct the problem of a functional integration measure. The interesting point here is that this functional can be chosen in different ways for the diagonal, $G^{i}$, and off-diagonal, $G^{a}$, sectors; i.e., it is precisely here that the color symmetry breaking takes place. In the case of the maximal Abelian gauge, the off-diagonal condition can be obtained through the extremization of the following auxiliary functional, ${ }^{13,14}$ :

\footnotetext{
${ }^{13}$ To impose the minimum condition it is necessary to take into account also $\delta^{2} H[A]>0$. This implies that the Faddeev-Popov operator, $\frac{\delta G\left[A^{\omega}\right]}{\delta \omega}$, must be positive definite. This extra condition also implies that the domain of the functional integral is restricted to a region called Gribov's region and its implementation is well defined only in Euclidean space [23,24].

${ }^{14}$ The Landau gauge can also be defined as an extreme condition of an auxiliary functional. In this case, the suitable functional is $\int d^{4} x A_{\mu}^{A} A^{A \mu}$.
}

$$
H[A]=\frac{1}{2} \int d^{4} x A_{\mu}^{a} A^{a \mu} .
$$

Applying the extreme condition,

$$
\delta H[A]=0,
$$

we have then

$$
G^{a}[A]=D_{\mu}^{a b} A^{b \mu}=0
$$

Since the symmetry subgroup $U(1)^{N-1}$ is present in the theory, it is also necessary to choose a gauge condition for the diagonal components. For simplicity, a Landau-like gauge condition is taken,

$$
G^{i}[A]=\partial_{\mu} A^{i \mu}=0 .
$$

Thus, the set of equations (A24) and (A25) forms the socalled maximal Abelian gauge. It is important to mention here that the diagonal condition does not follow from an extreme condition of any auxiliary functional and different choices could be taken for this sector. This is the case, e.g., for the modified maximal Abelian gauge [31].
[1] G. 't Hooft, Nucl. Phys. B190, 455 (1981).

[2] S. S. Gubser and A. Karch, Annu. Rev. Nucl. Part. Sci. 59, 145 (2009).

[3] M. A. L. Capri, H. C. Toledo, and J. A. Helayël-Neto, Phys. Rev. D 91, 125017 (2015).

[4] M. A. L. Capri, D. M. Van Egmond, M. S. Guimaraes, O. Holanda, S. P. Sorella, R. C. Terin, and H. C. Toledo, Eur. Phys. J. C 78, 797 (2018).

[5] C. Becchi, A. Rouet, and R. Stora, Ann. Phys. (N.Y.) 98, 287 (1976).

[6] O. Piguet and A. Rouet, Phys. Rep. 76, 1 (1981).

[7] O. Piguet and S. P. Sorella, Lect. Notes Phys., M: Monogr. 28, 1 (1995).

[8] T. E. Clark, O. Piguet, and K. Sibold, Ann. Phys. (N.Y.) 109, 418 (1977).

[9] O. Piguet and K. Sibold, Nucl. Phys. B197, 257 (1982).

[10] O. Piguet and K. Sibold, Nucl. Phys. B197, 272 (1982).

[11] O. Piguet and K. Sibold, Report No. CERN-TH-4690/87, DOI: $10.1007 / \mathrm{BFb} 0033712$.

[12] O. Piguet, arXiv:hep-th/9611003.

[13] M. A. L. Capri, D. Fiorentini, A. D. Pereira, and S. P. Sorella, Phys. Rev. D 96, 054022 (2017).

[14] M. A. L. Capri, D. M. van Egmond, G. Peruzzo, M. S. Guimaraes, O. Holanda, S. P. Sorella, R. C. Terin, and H. C. Toledo, Ann. Phys. (Amsterdam) 390, 214 (2018).
[15] S. J. Gates, M. T. Grisaru, M. Rocek, and W. Siegel, Front. Phys. 58, 1 (1983).

[16] D. Bailin and A. Love, Graduate Student Series in Physics (Hilger, Bristol, 1986), p. 348.

[17] A. Ali, C. Isham, T. Kibble, and Riazuddin, World Scientific Series in 20th Century Physics (World Scientific, Singapore, 1994), Vol. 5, p. 679.

[18] A. R. Fazio, V. E. R. Lemes, M. S. Sarandy, and S. P. Sorella, Phys. Rev. D 64, 085003 (2001).

[19] D. Dudal, J. A. Gracey, V. E. R. Lemes, M. S. Sarandy, R. F. Sobreiro, S. P. Sorella, and H. Verschelde, Phys. Rev. D 70, 114038 (2004).

[20] O. Piguet and S. P. Sorella, Phys. Lett. B 371, 238 (1996).

[21] O. Piguet and K. Sibold, Report No. UGVA-DPT1982/07359, http://cds.cern.ch/record/139565.

[22] M. A. L. Capri et al. (to be published).

[23] V. N. Gribov, Nucl. Phys. 139B, 1 (1978).

[24] N. Vandersickel and D. Zwanziger, Phys. Rep. 520, 175 (2012).

[25] M. M. Amaral, Y. E. Chifarelli, and V. E. R. Lemes, J. Phys. A 47, 075401 (2014).

[26] M. A. L. Capri, V. E. R. Lemes, R. F. Sobreiro, S. P. Sorella, and R. Thibes, Phys. Rev. D 72, 085021 (2005).

[27] M. A. L. Capri, V.E. R. Lemes, R. F. Sobreiro, S. P. Sorella, and R. Thibes, Phys. Rev. D 74, 105007 (2006). 
[28] M. A. L. Capri, V. E. R. Lemes, R. F. Sobreiro, S. P. Sorella, and R. Thibes, Phys. Rev. D 77, 105023 (2008).

[29] M. A. L. Capri, M. S. Guimaraes, V. E. R. Lemes, S. P. Sorella, and D. G. Tedesco, Ann. Phys. (Amsterdam) 344, 275 (2014).
[30] M. A. L. Capri, A. J. Gomez, M. S. Guimaraes, V. E. R. Lemes, and S. P. Sorella, J. Phys. A 43, 245402 (2010).

[31] D. Dudal, J. A. Gracey, V. E. R. Lemes, R. F. Sobreiro, S. P. Sorella, R. Thibes, and H. Verschelde, J. High Energy Phys. 07 (2005) 059. 